\title{
METRIC ENTROPY AND APPROXIMATION ${ }^{1}$
}

\author{
BY G. G. LORENTZ
}

1. Introduction. The notion of metric entropy (called also $\epsilon$ entropy) has been invented by Kolmogorov [16], [19] in order to classify compact metric sets according to their massivity. The basic definitions are as follows.

Let $A$ be a subset of a metric space $X$, and let $\epsilon>0$ be given. A family $U_{1}, \cdots, U_{n}$ of subsets of $X$ is an $\epsilon$-covering of $A$ if the diameter of each $U_{k}$ does not exceed $2 \epsilon$ and if the sets $U_{k}$ cover $A$. For a given $\epsilon>0$, the number $n$ depends upon the covering family, but $N_{\epsilon}(A)=\min n$ is an invariant of the set $A$. The logarithm

$$
H_{\epsilon}(A)=\log N_{\epsilon}(A)
$$

is the entropy of $A$. (Sometimes this definition is modified by assuming that the sets $U_{k}$ are balls of radius $\epsilon_{\text {.) }}$

Points $y_{1}, \cdots, y_{m}$ of $A$ are called $\epsilon$-distinguishable if the distance between each two of them exceeds $\epsilon$. The number $M_{\epsilon}(A)=\max m$ is an invariant of the set $A$, and

$$
C_{\epsilon}(A)=\log M_{\epsilon}(A)
$$

is called the capacity of $A$. The main general fact about $C_{\epsilon}(A)$ and $H_{\epsilon}(A)$ is the simple set of inequalities

$$
C_{2 \epsilon}(A) \leqq H_{\epsilon}(A) \leqq C_{\epsilon}(A) .
$$

In general, $C_{\epsilon}(A)$ and $H_{\epsilon}(A)$ increase rapidly to $+\infty$ as $\epsilon \rightarrow 0$; their asymptotic behavior serves to describe the compact set $A$.

For the computation of the entropy of concrete sets of functions, Kolmogorov [16], [19], Vituškin [37] and others, have used different special devices. The results obtained were mainly valid for the uniform metric, and for sets $A$, whose approximation properties by polynomials, or by arbitrary linear combinations of fixed functions were well known. More precisely, the sets $A$ under consideration were sets $A(\Delta, \Phi)$ described below, or at least approximable by such sets.

Let $\Phi=\left\{\phi_{1}, \cdots, \phi_{n}, \cdots\right\}$ be a fundamental sequence of points in

${ }^{1}$ Based on an address entitled Applications of entropy to approximation delivered by Professor Lorentz by invitation of the Committee to Select Hour Speakers for Summer and Annual Meetings, on Friday, September 3, 1965; received by the editors May 30, 1966. This work has been supported, in part, by the Contract no. AF 49 (638)-1401 of the Office of Scientific Research, U. S. Air Force. 
a Banach space $X$, that is, a sequence of points whose linear combinations are dense in $X$.

Let $\Delta=\left\{\delta_{0}, \cdots, \delta_{n}, \cdots\right\}$ be a sequence of numbers for which $\delta_{n}>0, \delta_{0} \geqq \delta_{1} \geqq \cdots, \delta_{n} \rightarrow 0$. The $n$th degree of approximation of an element $f \in X$ by the system $\Phi$ is given by

$$
E_{n}^{\Phi}(f)=\min _{\left\{a_{k}\right\}}\left\|f-\sum_{k=1}^{n} a_{k} \phi_{k}\right\|, \quad n=0,1, \cdots .
$$

The set $A(\Delta, \Phi)$ consists of all elements $f \in X$ which satisfy the inequalities $E_{n}^{\phi}(f) \leqq \delta_{n}, n=0,1, \cdots$. A set $A(\Delta, \Phi)$ is sufficiently rich, because by a theorem of S. Bernș̌teǐn (Davis $[8$, p. 332]), for each $\Delta$ there is an $f \in X$ for which $E_{n}^{\Phi}(f)=\delta_{n}, n=0,1, \cdots$ We shall call the sets $A(\Delta, \Phi)$ the full approximation sets.

Is it possible to develop a method for the evaluation of the entropy of each set $A(\Delta, \Phi)$ ? We shall give a positive answer to this question. It is remarkable that neither the structure of the sequence $\Phi$, nor the properties of the norm of the space $X$ are important for the final results. In this way we obtain a uniform derivation of known estimates (Kolmogorov's for the classes $\Lambda_{r, \alpha}$, Vituškin's for analytic functions) as well as new ones (for example, classes $\Lambda_{r, \alpha}^{p}$ in the $L^{p}$-norm).

As forerunners of our results we mention the computation of entropies of ellipses by Kolmogorov and Tihomirov [19, p. 40] and Mitjagin [25], and papers of Brudnyî and Timan [5] and of Helemskiî and Henkin [14], which deal with arbitrary compacts in Hilbert and Banach spaces.

For a general theory of entropy, the reader can consult the article [19] and the books of Vituškin [37] and Lorentz [24, Chapter 10].

The plan of this paper is as follows. In $\S 2$, we study some geometric properties of compacts in finitely and infinitely dimensional Banach spaces. In §3, our main results (Theorems 2 and 3) are established; they concern the entropy of full approximation sets. Their usefulness for the computation of entropies of concrete sets is illustrated in $\$ 5$. Another application, in $\S 4.2$, is to the "stability" of the approximation of full approximation sets. The estimate $E_{n}^{\Phi}(f) \leqq \delta_{n}$ will not be essentially improved for most $f \in A(\Delta, \Phi)$ if $\Phi$ is replaced by some other sequence $\Psi$, and even if one is allowed to select for each $f$ the most favorable from a countable set of sequences $\Psi_{1}, \Psi_{2}, \cdots$.

Another application of ideas of $\S 3$ is to theorems of Vituškin's type in Banach spaces: in $\S 4.1$ for linear approximation, in $\S 7$ to (piecewise) polynomial approximation. A very simple proof of Vituskin's theorem for rational approximation in uniform norm is offered in $\$ 6$. Finally, $\$ 5$ contains a review of recent results on entropy. 
2. Properties of ellipsoids and of full approximation sets. In this section we shall study the geometric and the measure-theoretic properties of certain sets. Let $X$ be a Banach space, $\Phi=\left\{\phi_{1}, \phi_{2}, \cdots\right\}$ a fundamental sequence in $X$. Let $X_{n}$ be the $n$-dimensional subspace of $X$ spanned by $\phi_{1}, \cdots, \phi_{n}$. To certain subsets $B$ of $X$ we assign their "euclidean volume" $|B|$, by identifying it with the volume of the image of $B$ in $R^{n}$ under the map $a_{1} \phi_{1}+\cdots+a_{n} \phi_{n} \rightarrow\left(a_{1}, \cdots, a_{n}\right)$. For $k \leqq n$, the volume in the subspace $X_{k}$ of $X_{n}$ (and in subspaces of $X_{n}$ parallel to $X_{k}$ ) will be denoted by $|B|_{k}$.

In $X_{n}$ we consider the unit ball $U$, balls $U_{r}$ with center origin and radius $r>0$, and "ellipsoids" $E=E\left(\delta_{0}, \cdots, \delta_{n-1}\right)$, which consist of all points $a_{1} \phi_{1}+\cdots+a_{n} \phi_{n}$ for which

$$
\frac{a_{1}}{\delta_{0}} \phi_{1}+\cdots+\frac{a_{n}}{\delta_{n-1}} \phi_{n} \in U \text {. }
$$

If $|U|=\lambda_{n}$, then $\left|U_{r}\right|=\lambda_{n} r^{n}$, and

$$
\left|E\left(\delta_{0}, \cdots, \delta_{n-1}\right)\right|=\lambda_{n} \delta_{0}, \cdots, \delta_{n-1} .
$$

A set $A=A\left(\delta_{0}, \cdots, \delta_{n-1}\right)$ is the set of all points $f \in X_{n}$ for which $E_{k}^{\Phi}(f) \leqq \delta_{k}, k=0,1, \cdots, n-1$.

LEMma 1. For the ball $U_{r}$ of $X_{n}$, and $0<\epsilon \leqq r$,

$$
\frac{1}{2^{n}}\left(\frac{r}{\epsilon}\right)^{n} \leqq N_{\epsilon}\left(U_{r}\right) \leqq 3^{n}\left(\frac{r}{\epsilon}\right)^{n} \text {. }
$$

Proof. Let $y_{1}, \cdots, y_{m}, m=M_{\epsilon}\left(U_{r}\right)$ be a maximal set of $\epsilon$-distinguishable points of $U_{r}$. The closed balls with centers $y_{i}$ and radii $\epsilon$ cover $U_{r}$. Comparing the euclidean volumes, we see that $\lambda_{n} \epsilon^{n} M_{\epsilon}\left(U_{r}\right)$ $\geqq \lambda_{n} r^{n}$.

On the other hand, balls with centers $y_{i}$ and radii $\epsilon / 2$ are disjoint, and contained in the ball $U_{r+\epsilon / 2} \subset U_{3 r / 2}$. It follows $\lambda_{n}(\epsilon / 2)^{n} M_{\epsilon}\left(U_{r}\right)$ $\leqq \lambda_{n}(3 r / 2)^{n}$. Thus,

$$
\left(\frac{r}{\epsilon}\right)^{n} \leqq M_{\epsilon}\left(U_{r}\right) \leqq 3^{n}\left(\frac{r}{\epsilon}\right)^{n} .
$$

Relation (2) follows from this and the inequalities $M_{2 \epsilon}(A) \leqq N_{\epsilon}(A)$ $\leqq M_{\epsilon}(A)$.

Lemma 2. Let $E$ be the ellipsoid $E\left(\delta_{0}, \cdots, \delta_{n-1}\right) \delta_{0} \geqq \cdots \geqq \delta_{n-1}>0$ in $X_{n}$, let $E_{a_{k}^{0} \ldots, a_{n}^{0}}$ be the $(k-1)$-dimensional section of $E$, given by the relations $a_{i}=a_{i}^{0}, i=k, \cdots, n$. If $|\lambda| \leqq 1$ and $a_{i}^{\prime}=\lambda a_{0}^{0}, i=k, \cdots, n$, then 


$$
\left|E_{a_{k}, \cdots, a_{n}^{\prime}}^{\prime}\right| \geqq\left|E_{a_{k}, \cdots, a_{n}}^{0}\right|
$$

Proof. We put

$$
\begin{gathered}
F=E_{a_{k}, \ldots, a_{n}}^{0}, \quad F_{-}=E_{-a_{k}, \cdots,-a_{n},}^{0}, \\
F^{\prime}=E_{a_{k}, \cdots, a_{n}}^{\prime}, \quad F^{\prime \prime}=\frac{1+\lambda}{2} F+\frac{1-\lambda}{2} F_{-} .
\end{gathered}
$$

The set $F_{-}$is obtained from $F$ by the mapping $x \rightarrow-x$ and $X_{n}$ onto itself, hence $\left|F_{-}\right|=|F|$. If $x=\left(a_{1}, \cdots, a_{n}\right) \in F^{\prime \prime}$, then

$$
a_{i}=\frac{1+\lambda}{2} a_{i}^{0}-\frac{1-\lambda}{2} a_{i}^{0}=\lambda a_{i}^{0}=a_{i}^{\prime}, \quad i=k, \cdots, n .
$$

Moreover, since $E$ is convex, $F^{\prime \prime} \subset E$. It follows that $F^{\prime \prime} \subset E_{a_{k}^{\prime}}, \ldots, a_{n}^{\prime}$ $=F^{\prime}$.

By the theorem of Brun-Minkowski about mixed volumes [3, p. 88],

$$
\left|F^{\prime \prime}\right| \geqq \frac{1+\lambda}{2}|F|+\frac{1-\lambda}{2}\left|F_{-}\right|=|F| \text {. }
$$

Hence $\left|F^{\prime}\right| \geqq|F|$.

Theorem 1. Let $E=E\left(\delta_{0}, \cdots, \delta_{n-1}\right)$ and $A=A\left(\delta_{0}, \cdots, \delta_{n-1}\right)$ be subsets of $X_{n}$. Then their Euclidean volumes satisfy the relation

$$
\left|E\left(\delta_{0}, \cdots, \delta_{n-1}\right)\right| \leqq\left|A\left(\delta_{0}, \cdots, \delta_{n-1}\right)\right| .
$$

Proof. The set $A$ consists of all points $x=a_{1} \phi_{1}+\cdots+a_{n} \phi_{n}$ $=\left(a_{1}, \cdots, a_{n}\right) \in X_{n}$ for which

$$
\min _{x_{1}, \cdots, x_{n-1}}\left\|x_{1} \phi_{1}+\cdots+x_{n-1} \phi_{n-1}+\frac{a_{n}}{\delta_{n-1}} \phi_{n}\right\| \leqq 1,
$$

$$
\begin{gathered}
\min _{x_{1}}\left\|x_{1} \phi_{1}+\frac{a_{2}}{\delta_{1}} \phi_{2}+\cdots+\frac{a_{n}}{\delta_{1}} \phi_{n}\right\| \leqq 1, \\
\left\|\frac{a_{1}}{\delta_{0}} \phi_{1}+\cdots+\frac{a_{n}}{\delta_{0}} \phi_{n}\right\| \leqq 1 ;
\end{gathered}
$$

while $E$ is given by one single inequality

$$
\left\|\frac{a_{1}}{\delta_{0}} \phi_{1}+\cdots+\frac{a_{n}}{\delta_{n-1}} \phi_{n}\right\| \leqq 1 .
$$


We introduce intermediate sets $A_{k}, k=1, \cdots, n-1$ in $X_{n}$. A point $\left(a_{1}, \cdots, a_{n}\right)$ belongs to $A_{k}$ if and only if

$$
\min _{x_{1}, \cdots, x_{n-1}}\left\|x_{1} \phi_{1}+\cdots+x_{n-1} \phi_{n-1}+\frac{a_{n}}{\delta_{n-1}} \phi_{n}\right\| \leqq 1
$$

(8)

$$
\begin{array}{r}
\min _{x_{1}, \cdots, x_{k}}\left\|x_{1} \phi_{1}+\cdots+x_{k} \phi_{k}+\frac{a_{k+1}}{\delta_{k}} \phi_{k+1}+\cdots+\frac{a_{n}}{\delta_{k}} \phi_{n}\right\| \\
\left\|\frac{a_{1}}{\delta_{0}} \phi_{1}+\cdots+\frac{a_{k-1}}{\delta_{k-2}} \phi_{k-1}+\frac{a_{k}}{\delta_{k-1}} \phi_{k}+\cdots+\frac{a_{n}}{\delta_{k-1}} \phi_{n}\right\| \leqq 1
\end{array}
$$

Conditions (8) contain $n-k+1$ inequalities. All but the last one disappear if $k=n$. Therefore, $A_{1}=A, A_{n}=E$. Hence all that we have to show is that $\left|A_{k}\right| \leqq\left|A_{k-1}\right|$ for $k=2, \cdots, n$. Let $G_{k}, k=2, \cdots, n$ be the $n$-k-dimensional region of the change of $a_{k+1}, \cdots, a_{n}$, given by the first $n-k$ inequalities (8). Then

$$
\begin{aligned}
& \left|A_{k}\right|=\int_{G_{k}}\left|E_{\delta_{k} a_{k+1} / \delta_{k-1}}, \cdots, \delta_{n-1} a_{n} / \delta_{k-1}\right| k d a_{k+1} \cdots d a_{n} \\
& =\int_{G_{k}} d a_{k+1} \cdots d a_{n} \int\left|E_{a_{k}, \delta_{k} a_{k+1} / \delta_{k-1}, \cdots, \delta_{n-1} a_{n} / \delta_{k-1}}\right|_{k-1} d a_{k},
\end{aligned}
$$

where the last integral is extended over all $a_{k}$ for which

$$
\min _{x_{1}, \cdots, x_{k-1}}\left\|x_{1} \phi_{1}+\cdots+x_{k-1} \phi_{k-1}+\frac{a_{k}}{\delta_{k-1}} \phi_{k}+\cdots+\frac{a_{n}}{\delta_{k-1}} \phi_{n}\right\| \leqq 1 .
$$

Applying Lemma 2, we obtain

$$
\left|A_{k}\right| \leqq \int_{G_{k-1}}\left|E_{\delta_{k-1} a_{k} / \delta_{k}}, \cdots, \delta_{n-1} a_{n} / \delta_{k}\right| k-1 d a_{k} \cdots d a_{n}=\left|A_{k-1}\right| .
$$

We now return to the full approximation sets $A=A(\Delta, \Phi)$ in separable Banach spaces $X$. It is easy to see that each set $A$ is compact. Their widths ([22], [33], [24]) can also be determined:

$$
d_{n}(A)=\delta_{n}, \quad n=0,1, \cdots .
$$

Indeed, from the definition of $A$ and the widths, $d_{n}(A) \leqq \delta_{n}$. On the other hand, $A_{n}=A\left(\delta_{0}, \cdots, \delta_{n-1}\right) \subset X_{n}$ contains the ball $U_{\delta_{n-1}}$ of $X_{n}$. By Tihomirov's theorem [33, p. 84], [24, p. 137], in the space $X$, $d_{n-1}\left(U_{\delta_{n-1}}\right)=\delta_{n-1}$. Hence 


$$
d_{n-1}(A) \geqq d_{n-1}\left(A_{n}\right) \geqq \delta_{n-1}, \quad n=1,2, \cdots .
$$

Lemma 3. Let $A(\Delta, \Phi)$ be a full approximation set in the Banach space $X$, let $A^{\prime}=A\left(\delta_{0}+2 \delta_{n}, \cdots, \delta_{n-1}+2 \delta_{n}\right)$ be a subset of $X_{n}$. Let $B$ be the full approximation set of the factor space $Y=X / X_{n}$, which corresponds to the sequences $\delta_{n}, \delta_{n+1}, \cdots$ and $\psi_{n}, \psi_{n+1}, \cdots$, where $\psi_{i}, i=n$, $n+1, \cdots$ is the factor class which contains $\phi_{i}$. If $\epsilon=\epsilon_{1}+\epsilon_{2}, \epsilon_{1}>0$, $\epsilon_{2}>0$, then

$$
H_{\epsilon}(A(\Delta, \Phi)) \leqq H_{\epsilon_{1}}\left(A^{\prime}\right)+H_{\epsilon_{2}}(B) .
$$

Proof. Let $B_{0}$ be the set of all $\eta \in Y$ for which $\eta \cap A(\Delta, \Phi) \neq 0$. Then

$$
B_{0} \subset B \text {. }
$$

Indeed, let $x \in \eta \cap A(\Delta) \neq 0$. Since $x \in A(\Delta)$, there exists for each $m \geqq n \quad$ a linear combination $a_{1} \phi_{1}+\cdots+a_{m} \phi_{m}$ for which $\left\|x-\left(a_{1} \phi_{1}+\cdots+a_{m} \phi_{m}\right)\right\| \leqq \delta_{m}$. This implies

$$
\left\|\eta-\left(a_{n} \psi_{n}+\cdots+a_{m} \psi_{m}\right)\right\|_{\boldsymbol{r}} \leqq \delta_{m}, m \geqq n \text {. }
$$

Hence $\eta \in B$.

Assume now that $A_{0}$ is the subset of $X$ that consists of all points $x$ with the property $E_{k}(x) \leqq \delta_{k}^{\prime}, k=0, \cdots, n-1$. Then for each class $\eta \in Y$, the set $\eta \cap A_{0}$ has an $\epsilon$-net containing at most $N_{\epsilon}\left(A\left(\delta_{0}^{\prime}+\delta_{n}^{\prime}\right.\right.$, $\left.\left.\cdots, \delta_{n-1}{ }^{\prime}+\delta_{n}{ }^{\prime}\right)\right)$ points.

For the proof, let $\eta \cap A_{0} \neq 0$. If $x \in \eta \cap A_{0}$, we have $\rho\left(x, X_{n}\right) \leqq \delta_{n}^{\prime}$. This implies that there exists a point $x_{0} \in \eta$ with $\left\|x_{0}\right\| \leqq \delta_{n}{ }^{\prime}$. Then $\eta=x_{0}+X_{n}$. The sets $\eta \cap A_{0}$ and $C=\eta \cap A_{0}-x_{0}$ are isometric, and it is sufficient to construct a required $\epsilon$-net for $C$ in $X_{n}$. However, if $y \in C$, then $y=x-x_{0}, x \in A_{0}$, and $E_{k}(y) \leqq \delta_{k}{ }^{\prime}+\left\|x_{0}\right\| \leqq \delta_{k}{ }^{\prime}+\delta_{n}{ }^{\prime}, k<n$. Therefore, $C \subset A\left(\delta_{0}^{\prime}+\delta_{n}^{\prime}, \cdots, \delta_{n-1}^{\prime}+\delta_{n}^{\prime}\right)$, and the statement follows.

We can now prove (10). Let $\epsilon_{2}<\delta_{n}$, let $\eta_{1}, \cdots, \eta_{m}, m=N_{\epsilon_{2}}\left(B_{0}\right)$ be an $\epsilon_{2}$-net for $B_{0}$. For each $i$, let $x_{i j}, j=1, \cdots, N_{\epsilon_{1}}\left(\eta_{i} \cap A_{0}\right)$ be an $\epsilon_{1}$-net for the set $\eta_{i} \cap A_{0}$, where $A_{0}$, with $\delta_{k}^{\prime}=\delta_{k}+\delta_{n}, k=0, \cdots, n-1$, has been described above. Then the $x_{i j}$ form an $\epsilon$-net for $A(\Delta)$. In fact, let $y \in A(\Delta)$, and let $\eta$ be the class to which $y$ belongs. Then for some $i,\left\|\eta-\eta_{i}\right\| \leqq \epsilon_{2}$, and we can find a $y_{i} \in \eta_{i}$ for which $\left\|y-y_{i}\right\| \leqq \epsilon_{2}\left(<\delta_{n}\right)$. Then $E_{k}\left(y_{i}\right) \leqq \delta_{k}+\delta_{n}=\delta_{k}^{\prime}, k=0, \cdots, n-1$; hence $y_{i} \in \eta_{i} \cap A_{0}$. Then for some $j,\left\|y-x_{i j}\right\| \leqq \epsilon$.

The number of points $x_{i j}$ does not exceed

$$
N_{\epsilon_{1}}\left(A\left(\delta_{0}^{\prime}+\delta_{n}^{\prime}, \cdots, \delta_{n-1}^{\prime}+\delta_{n}^{\prime}\right)\right) m,
$$

and we obtain (10).

The case $\epsilon_{2} \geqq \delta_{n}$ is simpler. Here $B_{0}$ is contained in the ball $U_{\epsilon_{2}}$ of 
the space $Y$, and the set $\eta_{1}, \cdots, \eta_{m}$ can be replaced by the zero element of $Y$.

Remark. The spaces $X$ and $X_{n}$ have been real Banach spaces. Similar considerations hold for complex Banach spaces. In this case, $X_{n}$ is the set of all linear combinations $\alpha_{1} \phi_{1}+\cdots+\alpha_{n} \phi_{n}$ with complex $\alpha_{k}$ or of all real linear combinations of $2 n$ elements $\phi_{k}, i \phi_{k}$, $k=1, \cdots, n$. In this case, $n$ should be replaced by $2 n$ in the formula (2), while instead of (1) we have $\left|E\left(\delta_{1}, \cdots, \delta_{n}\right)\right|=\lambda_{n} \delta_{1}^{2}, \cdots, \delta_{n}^{2}$. No changes are necessary in Lemmas 2,3 and Theorem 1 .

3. The main results. Let $A=A(\Delta, \Phi)$ be a full approximation set in a Banach space $X$. Let $C>1$ be a fixed constant. We define

$$
N_{0}=0, N_{i}=\min \left\{k: \delta_{k} \leqq C^{-i}\right\}, \quad i=1,2, \cdots .
$$

The sequence $N_{i}$ increases to $+\infty$, and

(2) $C^{-(i+1)}<\delta_{k} \leqq C^{-i} \quad$ if $\quad N_{i} \leqq k<N_{i+1}, \quad i=1,2, \cdots$.

Let $\Delta N_{i}=N_{i+1}-N_{i}, i=0,1, \cdots$.

Theorem 2. For a given $\epsilon>0, \epsilon \leqq 1$, let $j$ be defined by

$$
C^{-(j-1)}<\epsilon \leqq C^{-(j-2)} \text {. }
$$

Then

(5) $H_{\epsilon}(A) \leqq\left(N_{1}+\cdots+N_{j}\right) \log C+$

$$
N_{j} \log \frac{9}{C-1}+\sum_{i=0}^{j-1} N_{i} \log \frac{N_{j}}{\Delta N_{i}}+N_{1} \log \delta_{0} .
$$

Proof. We begin with (4). Let $n$ be arbitrary, and let $A_{n}=A \cap X_{n}$. Let $y_{1}, \cdots, y_{m}, m=M_{\epsilon}\left(A_{n}\right)$, be a maximal set of $\epsilon$-distinguishable points in $A_{n}$. The closed balls of $X_{n}$ with centers $y_{i}$ and radii $\epsilon$ cover $A_{n}$. Therefore $m \lambda_{n} \epsilon^{n} \geqq\left|A_{n}\right|$. By Theorem 1 and 2(1) we obtain

$$
M_{\epsilon}(A) \geqq M_{\epsilon}\left(A_{n}\right) \geqq \frac{\delta_{0} \cdots \delta_{n-1}}{\epsilon^{n}}, C_{\epsilon}(A) \geqq \sum_{k=0}^{n-1} \log \frac{\delta_{k}}{\epsilon} .
$$

We take $n=N_{j-2}$. By (2) and (3),

$$
\begin{aligned}
C_{\epsilon}(A) & \geqq \sum_{i=1}^{j-3} \sum_{N_{i} \leq k<N_{i+1}} \log \frac{\delta_{k}}{\epsilon} \\
& \geqq \sum_{i=1}^{j-3} \Delta N_{i} \log \left(C^{-i-1} C^{j-2}\right)=\sum_{i=1}^{j-3}(j-i-3) \Delta N_{i} \log C \\
& =\left(N_{1}+N_{2}+\cdots+N_{j-3}\right) \log C .
\end{aligned}
$$


To prove (5), we apply several times Lemma 3 . Let $\epsilon_{i}>0$, and let $\sum_{0}^{j-1} \epsilon_{i}=(1-1 / C) \epsilon$. We have

$$
H_{\epsilon}(A) \leqq \sum_{i=0}^{j-1} H_{\epsilon_{i}}\left(B_{i}\right)+H_{\epsilon / C}(B)
$$

where $B$ is the set $A\left(\delta_{N_{j}}, \delta_{N_{j+1}}, \cdots\right)$ in some Banach space $Y$, while $B_{i}$ is the set $A\left(3 \delta_{N_{i}}, \cdots, 3 \delta_{N_{i+1}-1}\right)$ in some $\Delta N_{i}$-dimensional space $Y_{i}$. [If $\Delta N_{i}=0$ for some $i$, then the space $Y_{i}$ is 0 -dimensional, and $H_{\delta}\left(B_{i}\right)=0$ for each $\delta>0$. For these $i$, we shall interpret the terms of the sum in (6), and of the last sum in (5) to be zero, and also put $\epsilon_{i}=0$.] By (2) and (3), $\delta_{N_{j}}<\epsilon / C$, and so $B$ is contained in the ball $U_{\epsilon / C}$ of the space $Y$. Hence $H_{\epsilon / C}(B)=0$. On the other hand, $B_{i}$ is contained in the ball $U_{r_{i}}$ of $Y_{i}$ with $r_{i}=3 \delta_{N_{i}}$. We estimate $H_{\epsilon_{i}}\left(U_{r_{i}}\right)$ by means of 2(2) and obtain

$$
H_{\epsilon}(A) \leqq \sum_{i=0}^{j-1} \Delta N_{i} \log \left(9 \delta_{N_{i}} / \epsilon_{i}\right) .
$$

We can select

$$
\epsilon_{i}=(1-1 / C) \epsilon \Delta N_{i} / N_{j} \quad i=0,1, \cdots, j-1,
$$

then

$$
\begin{aligned}
H_{\epsilon}(A) \leqq \sum_{i=0}^{j-1} \Delta N_{i} \log \left(\frac{9}{C-1} \frac{\delta_{N_{i}} N_{j}}{\Delta N_{i}} C^{j}\right) \\
\leqq N_{1} \log \delta_{0}+\left\{\Delta N_{0} \log \frac{9 C^{j}}{C-1}+\sum_{i=1}^{j-1} \Delta N_{i} \log \frac{9 C^{j-i}}{C-1}\right\} \\
+\sum_{i=0}^{j-1} \Delta N_{i} \log \frac{N_{j}}{\Delta N_{i}} .
\end{aligned}
$$

According to (2), the expression in the braces does not exceed

$$
\begin{aligned}
\sum_{i=0}^{j-1} \Delta N_{i} \log \frac{9 C^{j-i}}{C-1} & \leqq N_{j} \log \frac{9}{C-1}+\sum_{i=0}^{j-1}(j-i) \Delta N_{i} \log C \\
& =\left(N_{1}+\cdots+N_{j}\right) \log C+N_{j} \log \frac{9}{C-1},
\end{aligned}
$$

and (5) follows.

In many cases, we can put $C=e$ in (4) and (5); then we have

$$
\begin{aligned}
N_{1}+\cdots+N_{j-3} \leqq H_{\epsilon}(A) \leqq N_{1} & +\cdots+N_{j}+2 N_{j} \\
& +\sum_{i=0}^{j-1} N_{i} \log \frac{N_{j}}{\Delta N_{i}}+N_{1} \log \delta_{0}
\end{aligned}
$$


In other cases, it is necessary to take $C$ sufficiently close to 1 , so that the $N_{i}$ form a fairly dense net.

Theorem 2 should be compared with weaker results of Brudnyi and Timan [5]. Their Theorem 1 reads in our notations (but with logarithms in the definition of $H_{\epsilon}(A)$ to the base 2)

$$
H_{\epsilon}(A) \geqq n+1 \quad \text { if } \quad 0<\epsilon<\frac{1}{4} \delta_{n-1} .
$$

This is a weaker version of (4); for if in (1) and (3) $C=2$, one obtains $N_{j-3} \geqq n$. In the opposite direction these authors have [35, p. 392], [5, Theorem 7]:

$$
H_{e^{2} \epsilon}(A) \leqq\left(N_{j}+1\right) \log \frac{e^{2} \delta_{0}}{\epsilon}+\left(N_{j}+1\right) \log \left(N_{j}+2\right) .
$$

An inequality of this type follows from (5). In fact, we have

$$
N_{1}+\cdots+N_{j} \leqq j N_{j} \leqq\left(\log \frac{1}{\epsilon}+2\right) N_{j} .
$$

We now turn our attention to some more or less precise asymptotic formulas for $H_{\epsilon}(A)$, which can be derived from Theorem 2 for special classes of sequences $\delta_{n}$.

Lemma 4. One has

$$
S_{j}=\sum_{i=0}^{j-1} \Delta N_{i} \log \frac{N_{j}}{\Delta N_{i}} \leqq N_{1}+\cdots+N_{j}, \quad j=1,2, \cdots .
$$

For the proof we note that, if $S_{0}=0$, then for $k=1,2, \cdots$,

$$
\begin{aligned}
S_{k}-S_{k-1} & =\Delta N_{k-1} \log \frac{N_{k}}{\Delta N_{k-1}}+\sum_{i=0}^{k-2} \Delta N_{i} \log \frac{N_{k}}{N_{k-1}} \\
& =\left(N_{k}-N_{k-1}\right) \log \frac{N_{k}}{N_{k}-N_{k-1}}+N_{k-1} \log \frac{N_{k}}{N_{k-1}} .
\end{aligned}
$$

Now the function $f(x)=(b-x) \log (b /(b-x))+x \log (b / x)$ has its maximum on $[0, b]$ equal to $b \log 2<b$. This proves that $S_{k}-S_{k-1} \leqq N_{k}$, hence the inequality (11).

Lemma 5. Let $C=e$ in (1) and (3). (i) If

$$
\delta_{2 n} \leqq c \delta_{n}, \quad n=0,1, \cdots \text { for some } 0<c<1 \text {, }
$$

then

$$
N_{i+1} \leqq \text { Const. } N_{i} \text {. }
$$


(ii) If

$$
\frac{\delta_{[1+\epsilon) n]}}{\delta_{n}} \rightarrow 0 \text { as } n \rightarrow \infty \text { for each } \epsilon>0,
$$

then

$$
\begin{aligned}
\frac{N_{i+1}}{N_{i}} & \rightarrow 1, \\
S_{j}=\sum_{i=1}^{j-1} \Delta N_{i} \log \frac{N_{j}}{\Delta N_{i}} & =o\left(N_{1}+\cdots+N_{j}\right) .
\end{aligned}
$$

Proof. (i) Let $p$ be a natural number such that $c^{p} \leqq e^{-1}$. We have, by (13),

$$
\begin{aligned}
N_{i} & =\min \left\{k: \delta_{k} \leqq e^{-i}\right\} \geqq \min \left\{k: \delta_{2 k} \leqq c e^{-i}\right\} \\
& =\frac{1}{2} \min \left\{2 k: \delta_{2 k} \leqq c e^{-i}\right\} \geqq \frac{1}{2} \min \left\{k: \delta_{k} \leqq c e^{-i}\right\} .
\end{aligned}
$$

Repeating this, we obtain

$$
N_{i} \geqq \frac{1}{2^{p}} \min \left\{k: \delta_{k} \leqq c^{p} e^{-i}\right\} \geqq \frac{1}{2^{p}} N_{i+1} .
$$

(ii) Let $\epsilon>0$ be given. For all sufficiently large $i$, the inequality $\delta_{k} \leqq e^{-i}$ implies that $\delta_{[(1+\epsilon) k]} / \delta_{k} \leqq e^{-1}$. For all such $i$,

$$
\begin{aligned}
N_{i} & =\min \left\{k: e^{-1} \delta_{k} \leqq e^{-i-1}\right\} \geqq \min \left\{k: \delta_{[(1+\epsilon) k]} \leqq e^{-i-1}\right\} \\
& \geqq \frac{1}{1+\epsilon} \min \left\{[(1+\epsilon) k]: \delta_{[(1+\epsilon) k]} \leqq e^{-i-1}\right\} \\
& \geqq \frac{1}{1+\epsilon} N_{i+1} .
\end{aligned}
$$

Hence for all sufficiently large $i, 1 \leqq N_{i+1} / N_{i} \leqq 1+\epsilon$, and (16) is established.

From (12), with $x_{k}=N_{k-1} / N_{k}$ we have

$$
S_{k}-S_{k-1}=N_{k}\left\{\left(1-x_{k}\right) \log \frac{1}{1-x_{k}}+x_{k} \log \frac{1}{x_{k}}\right\} \text {. }
$$

For large $k, 0 \leqq 1-x_{k} \leqq \epsilon$. The functions $(1-x) \log (1 / 1-x)$ and $x \log (1 / x)$ approach zero for $x \rightarrow 0+$. Therefore, for all large $k$, the expression in the braces is $\leqq \epsilon_{1}$, where $\epsilon_{1}>0$ is arbitrarily small. Thus $S_{k}-S_{k-1}=o\left(N_{k}\right)$. Therefore we obtain (7). 
Theorem 3. Let $C=e$. Then

$$
H_{\epsilon}(A)=N_{1}+\cdots+N_{j}+O\left(S_{j}\right)
$$

If $\delta_{n}$ satisfies (13), then

$$
H_{\epsilon}(A) \approx N_{1}+\cdots+N_{j}
$$

If $\delta_{n}$ satisfies (15), then

$$
H_{\epsilon}(A) \sim N_{1}+\cdots+N_{j} .
$$

Proof. (18) follows from (10) and the inequality $N_{j} \leqq S_{j}$; (19) and (20) follow from (10) and Lemmas 4 and 5. More generally, we have (19) whenever the $N_{i}$ satisfy $N_{i+1}=O\left(N_{1}+\cdots+N_{i}\right)$.

Theorem 3 applies, roughly, to sequences $\delta_{n}$ that decrease to zero as $n^{\alpha}, \alpha>0$, or faster. The counterpart, Theorem 5 , will deal with sequences $\delta_{n}$ that decrease to zero slower than $n^{\alpha}, \alpha>0$. For the applications in $\$ 4.2$, however, we insert its more complicated version, Theorem 4.

Lemma 6. (i) If, for some $0<c<1$,

$$
\delta_{2 n} \geqq c \delta_{n},
$$

then, for the $N_{i}$ defined by (1) with $C=c^{-1}$,

$$
N_{i+1} \geqq 2 N_{i}-1 \text {. }
$$

(ii) If

$$
\lim _{n \rightarrow \infty} \frac{\delta_{2 n}}{\delta_{n}}=1,
$$

then for each $C>1$,

$$
\lim _{i \rightarrow \infty} \frac{N_{i+1}}{N_{i}}=+\infty
$$

Proof. We prove (ii); (i) is simpler. Let $C>1$ and a large integer $p$ be given; we define $c, 0<c<1$ by $c^{p}=C^{-1}$. For all large $k, \delta_{2 k} \geqq c \delta_{k}$, hence for all large $i$

$$
\begin{aligned}
N_{i} & \leqq \min \left\{k: c^{-1} \delta_{2 k} \leqq C^{-i}\right\} \\
& \leqq \frac{1}{2} \min \left\{k: \delta_{k} \leqq c C^{-i}\right\}+\frac{1}{2} .
\end{aligned}
$$

Repeating this $p$ times, we obtain

$$
N_{i} \leqq \frac{1}{2^{p}} \min \left\{k: \delta_{k} \leqq c^{p} C^{-i}\right\}+1 \leqq \frac{1}{2^{p}} N_{i+1}+1
$$


A statement weaker than (22) has been derived by Brudnyì and Timan [5, Theorem 2] from the assumption that $\delta_{n[\log n]} \geqq c \delta_{n}$.

The following theorem compares the entropies of two full approximation sets $A=A(\Delta, \Phi)$ and $A^{\prime}=A\left(\Delta^{\prime}, \Psi\right)$ if $\delta_{n}$ decreases slowly.

TheOREM 4. (i) Assume that $\delta_{n}$ satisfies (21) and that $\delta_{n}^{\prime} \leqq \delta_{n}$ for all large $n$. If $0<q<1$, then there is a $c_{1}>1$ (which depends only on $q$ and c) such that for all small $\epsilon>0$,

$$
H_{c_{1} \epsilon}\left(A^{\prime}\right) \leqq q H_{\epsilon}(A) .
$$

(ii) If the sequence $\delta_{n}$ satisfies (23) and if $\delta_{n}^{\prime} \leqq \delta_{n}$ for large $n$, then for each $c_{1}>1$,

$$
\lim _{\epsilon \rightarrow 0}\left\{H_{c_{1} \epsilon}\left(A^{\prime}\right) / H_{\epsilon}(A)\right\}=0 .
$$

Proof. We shall establish (i); the proof of (ii) is simpler. We take $C=c^{-1}$. Let $r$ be a large integer, to be fixed later. We take $c_{1} \geqq C^{4+r}$. If $j_{1}$ corresponds to $\epsilon_{1}=c_{1} \epsilon$ according to (3), then

$$
C^{-(j-1)}<\epsilon \leqq c_{1}^{-1} C^{-\left(j_{1}-2\right)} \leqq C^{-2-r-j_{1}},
$$

hence

$$
j_{1} \leqq j-r-3 \text {. }
$$

Let $N_{i}$ and $N_{i}^{\prime}$ denote the numbers (1) that correspond to the sets $A$ and $A^{\prime}$. By (5), (11), (27), (22) and (4), if $C_{1}=1+\log (9 C /(C-1))$,

$$
\begin{aligned}
H_{\epsilon_{1} c}\left(A^{\prime}\right) & \leqq O(1)+(1+\log C)\left(N_{1}^{\prime}+\cdots+N_{j_{1}}^{\prime}\right)+N_{j_{1}}^{\prime} \log \frac{9}{C-1} \\
& \leqq O(1)+\left(1+\log \frac{9 C}{C-1}\right)\left(N_{1}^{\prime}+\cdots+N_{j_{1}}^{\prime}\right) \\
& \leqq O(1)+C_{1}\left(N_{1}+\cdots+N_{j-r-3}\right) \\
& \leqq O(j)+2^{-r} C_{1}\left(N_{1}+\cdots+N_{j-3}\right) \\
& \leqq \frac{o(1)+2^{-r} C_{1}}{\log C} H_{\epsilon}(A) \leqq q H_{\epsilon}(A),
\end{aligned}
$$

if $r$ is selected large enough.

From Theorem 5 one can obtain relations that describe the behavior $H_{\epsilon}(A)$ for slowly decreasing $\delta_{n}$. Taking $A^{\prime}=A$, we have:

THEOREM 5. If the sequence $\delta_{n}$ satisfies (21), then for each $0<q<1$ and properly chosen $c_{1}>1$,

$$
H_{c_{1 \epsilon}}(A) \leqq q H_{\epsilon}(A) .
$$


If the sequence $\delta_{n}$ satisfies (23), then for each $c_{1}>1$,

$$
\lim _{\epsilon \rightarrow 0}\left\{H_{c_{1 \epsilon}}(A) / H_{\epsilon}(A)\right\}=0 .
$$

REMARK. Similar statements hold for sets $A(\Delta)$ in complex Banach spaces. However (compare $\$ 2$, Remark) one has then to replace the $N_{i}$ in the formulas (4), (5), (11) by $2 N_{i}$.

\section{Applications of main theorems.}

4.1. Results of Vituskin's type for linear approximation. Vituškin [37] has proved several theorems which provide lower bounds for the degree of approximation for some sets $A$ of functions. They apply to general, not necessarily linear, approximation (for example, to rational approximation), and will be discussed in $\$ \$ 6$ and 7 . For the linear approximation, his results reduce to statements of the following type. If for some $n$ and some $\epsilon>0$, the $n$th width (for the definition and properties of widths see [24, Chapter 9], [33]) of $A$ satisfies $d_{n}(A)<\epsilon$, then $n$ is at least as large as some simple lower bound, that depends on $\epsilon$, and is, roughly, $H_{\epsilon}(A)$.

In this section we deduce results of this type from Theorem 2 . They will be better than the special cases of Vituškin's theorems, mainly because our sets $A$ are arbitrary, or restricted by the asymptotic behavior of $H_{\epsilon}(A)$, while Vituškin restricts the structure of the class $A$.

THEOREM 6. Let $A$ be an arbitrary compact set in a separable Banach space $X$, and let $d_{n}(A)<\epsilon$. Then

$$
n \geqq \frac{H_{e^{2} \epsilon}(A)-C_{0}}{2+\log \frac{1}{\epsilon}+\log \left[H_{e^{2} \epsilon}(A)-C_{0}\right]} .
$$

If, in particular,

$$
H_{2 \epsilon}(A) / H_{\epsilon}(A) \rightarrow 1 \text { as } \epsilon \rightarrow 0,
$$

then $d_{n}(A)<\epsilon$ implies

$$
n \geqq \frac{(1-o(1)) H_{\epsilon}(A)}{\log \frac{1}{\epsilon}} .
$$

If for each $q, 0<q<1$ there is a $c_{1}>0$ for which $H_{c_{1} \epsilon}(A) \leqq q H_{\epsilon}(A)$, then $d_{n}(A)<\epsilon$ implies

$$
n \geqq C_{1} H_{e^{2} \epsilon}(A)-C_{2} \text {. }
$$


Proof. There exists a fundamental sequence $\Phi$ in $X$, for which $d_{n}(A) \leqq E_{n}^{\Phi}(A)<\epsilon$. Let $\delta_{k}=E_{k}^{\Phi}(A), \quad k=0,1, \cdots$, then $A \subset A^{\prime}$ $=A(\Delta, \Phi)$. In view of the monotonicity of the right-hand side of (1), it is sufficient to prove that if $\delta_{n} \leqq \epsilon$, then $n$ is not less than the righthand side of (1) with $A$ replaced by $A^{\prime}$. We take $C=e$ in 3(1) and 3(3), and have by $3(10)$

$$
H_{e^{2} \epsilon}\left(A^{\prime}\right)-N_{1} \log \delta_{0} \leqq N_{1}+\cdots+N_{j}+2 N_{j}+N_{j} \log N_{j}
$$

where $j$ is defined by $e^{-j-1}<\epsilon \leqq e^{-j}$. Since $\delta_{n} \leqq e^{-j}$, we have $n \geqq N_{j}$. Hence

$$
H_{e^{2} \epsilon}\left(A^{\prime}\right)-C_{0} \leqq(j+2+\log n) n \leqq(\log (1 / \epsilon)+2+\log n) n .
$$

Now we use the simple fact that if $\alpha \leqq n(\beta+\log n)$, where $\alpha>0, \beta>0$, $\beta+\log \alpha>1$, then $n>\alpha /(\beta+\log \alpha)$. This proves (1). Relation (3) is a special case, since (2) implies that

$$
\log H_{\epsilon}(A)=o\left(\log \frac{1}{\epsilon}\right) .
$$

To prove (4), we derive from $3(10)$ and $3(11)$

$$
H_{e^{2} \varepsilon}\left(A^{\prime}\right)-C_{0} \leqq 4\left(N_{1}+\cdots+N_{j}\right) .
$$

By 3(10) and the assumption with $q=1 / 5$ we have, if $r$ is sufficiently large,

$$
N_{1}+\cdots+N_{j-r-1} \leqq H_{e^{r} \epsilon}\left(A^{\prime}\right) \leqq \frac{1}{5} H_{e^{2} \epsilon}\left(A^{\prime}\right) .
$$

Therefore (6) yields

$$
\frac{1}{5} H_{e^{2} \epsilon}\left(A^{\prime}\right)-C_{0} \leqq 4(r+1) n,
$$

and (4) follows.

The result (3) should be compared with Vituškin's [37, p. 177, Theorem 1], where this inequality is proved for classes of analytic functions, and (4) with his [37, p. 182, Theorem 1], which holds for classes of "smooth" functions, such as $\Lambda_{p, \omega}^{s}$ (see $\S 5$ ). Results of Brudnyi and Timan [5, Theorems 3 and 5] are very special cases of (1), for compacts in a Hilbert space.

4.2. Comparison of sets $A(\Delta, \Phi)$ for different $\Delta$. The purpose of this section is to show that the set $A(\Delta, \Phi)$ decreases drastically if the numbers $\delta_{n}$ are replaced by smaller numbers $\delta_{n}{ }^{\prime}$, even if $\Phi$ is replaced by another set $\Psi$ in this process. For the sets $A=A(\Delta, \Phi)$ and $A^{\prime}$ $=A\left(\Delta^{\prime}, \Psi\right)$ we prove that $H_{\epsilon}\left(A^{\prime}\right)$ is asymptotically smaller than $H_{\epsilon}(A)$. In this context, entropy plays a role that is usually reserved for measure, category or cardinality in theorems about the compar- 
ison of sets. Results of this type allow us to establish the existence of elements $f \in X$, for which the $\Psi$-approximation is not essentially better than the $\Phi$-approximation.

Theorem 7. Let $A=A(\Delta, \Phi)$ and $A^{\prime}=A\left(\Delta^{\prime}, \Psi\right)$ be two full approximation sets in a Banach space $X$. (i) If

$$
\delta_{2 n} \geqq c \delta_{n}, \quad n=1,2, \cdots c>0,
$$

and if $0<q<1$, then there is a $q_{1}>0$ (which depends only on $c$ and $q$ ) with the property that $\lim \sup \left(\delta_{n}^{\prime} / \delta_{n}\right)<q_{1}$ implies

$$
\limsup _{\epsilon \rightarrow 0}\left\{H_{\mathbf{\epsilon}}\left(A^{\prime}\right) / H_{\mathbf{\epsilon}}(A)\right\}<q ;
$$

(ii) If $\delta_{n}$ satisfies (8) and if $\lim \left(\delta_{n}^{\prime} / \delta_{n}\right)=0$, then

$$
\lim _{\epsilon \rightarrow 0}\left\{H_{\epsilon}\left(A^{\prime}\right) / H_{\epsilon}(A)\right\}>0 \text {; }
$$

(iii) Relation (10) holds also if $\lim \sup \left(\delta_{n}^{\prime} / \delta_{n}\right)<q_{1}, q_{1}<1$ and if

$$
\lim _{n \rightarrow \infty}\left(\delta_{2 n} / \delta_{n}\right)=1 \text {. }
$$

Proof (i). Take $c_{1}$ from Theorem 4 (i), and put $q_{1}=c_{1}^{-1}$. Then $\delta_{n}^{\prime \prime}=\delta_{n}^{\prime} / q_{1} \leqq \delta_{n}$ for all large $n$. According to Theorem 4 (i) we have $H_{c_{1 \epsilon}}\left(A\left(\Delta^{\prime \prime}\right)\right) \leqq q H_{\epsilon}(A)$, for all small $\epsilon>0$. But $H_{c_{1 \epsilon}}\left(A\left(\Delta^{\prime \prime}\right)\right)=H_{\epsilon}\left(A\left(\Delta^{\prime}\right)\right)$. Statement (ii) follows from (i), while (iii) can be proved in a like manner, with the help of Theorem 5 (ii).

We shall use Theorem 7 to show that even an introduction of countably many sequences $\Psi_{p}$ instead of $\Phi$ cannot essentially reduce the degree of approximation of the elements $f \in A(\Delta, \Phi)$.

Theorem 8. Let $\Phi, \Psi_{p}, p=1,2, \cdots$ be fundamental sequences in $X$. Assume that the sequence $\delta_{n}$ satisfies (8), and let

$$
\lim \sup \delta_{n}^{(p)}=0, \quad p=1,2, \cdots, \delta_{n} .
$$

Then there exists an element $f \in X$ such that

$$
E_{n}^{\Phi}(f) \leqq \delta_{n}, \quad n=0,1, \cdots
$$

but the condition

$$
E_{n}^{\Psi p}(f) \leqq \delta_{n}^{(p)}, \quad n=0,1, \cdots
$$

is violated for each $p$. 
Proof. Let $Y$ be the set of all $f \in X$ with the property that $E_{n}^{\Phi}(f)$ $=O\left(\delta_{n}\right)$, and the norm $\rho(f)=\sup _{n}\left\{E_{n}^{\phi}(f) / \delta_{n}\right\}$. Standard arguments show that $Y$ is a Banach space with this norm. Let also $V_{p}, p=1$, $2, \cdots$ be the set of $f \in Y$ for which $E_{n}^{\Psi p}(f) \leqq \delta_{n}^{(p)}, n=0,1,2, \cdots$.

Now $E_{n}^{\Psi p}(f)$ is a continuous function of $f \in Y$ in the norm $\rho$, for $\rho\left(f_{s}-f\right) \rightarrow 0, s \rightarrow+\infty$ implies $\left\|f_{s}-f\right\| \rightarrow 0$, and $E_{n}^{\Psi p}(f)$ is continuous on $X$. Hence each $V_{p}$ is a closed subset of $Y$. From Theorem 7 (ii) it follows that $H_{\epsilon}\left(V_{p}\right)<H_{\epsilon}(U)$, for all small $\epsilon>0$, for each ball $U$ in $Y$ (with an arbitrary center and radius). Thus, $V_{p}$ does not exhaust $U$. In other words, $V_{p}$ is nowhere dense in $Y$. It follows, by Baire's theorem, that $Y-\cup_{p} V_{p} \neq 0$.

THEOREM 9. Assume that $\delta_{n}$ satisfies (8). There is a $\rho>0$ with the property that for each two fundamental sequences $\Phi, \Psi$, there is an element $f \in X$ which satisfies (13) and the inequality

$$
E_{n}^{\Psi}(f) \geqq \rho \delta_{n} \quad \text { for infinitely many } n .
$$

Proof. We select $\rho, 0<\rho<1$ so that $q_{1}=4 \rho / c$ works in Theorem 7 (i) for some $q<1$. By induction we define increasing sequences of integers $n_{i}, m_{i}, i=1,2, \cdots\left(m_{0}=0\right)$, and elements $f_{i}$ such that

$$
m_{i}>2 i, m_{i} \leqq n_{i}<m_{i+1}, i=1,2, \cdots ; \delta_{m_{k}} \leqq \frac{\rho}{2^{k-i}} \delta_{m_{i}} \text { for } i<k .
$$

The $(k+1)$ st step of the induction is as follows. We define two sequences of positive numbers $\Delta_{k+1}, \bar{\Delta}_{k+1}$, putting

$$
\begin{array}{rlrl}
\delta_{n}^{(k+1)} & =\frac{1}{2} \delta_{m_{k}} \text { for } n \leqq m_{k}, \bar{\delta}_{n}^{(k+1)} & =\delta_{0} \text { for } n<k+m_{k}, \\
& =\frac{1}{2} \delta_{n} \text { for } n>m_{k}, & & =2 \rho \delta_{n-k} \text { for } n \geqq k+m_{k} .
\end{array}
$$

We take $\Psi_{k+1}=\left\{f_{1}, \cdots f_{k}, \psi_{0}, \psi_{1}, \cdots\right\}$. Then

$$
\limsup _{n \rightarrow \infty} \frac{\bar{\delta}_{n}^{(k+1)}}{\delta_{n}^{(k+1)}}=\limsup _{n \rightarrow \infty} \frac{2 \rho \delta_{n-k}}{\frac{1}{2} \delta_{n}} \leqq \limsup _{n \rightarrow \infty} \frac{4 \rho \delta_{n}}{\delta_{2 n}} \leqq \frac{4 \delta}{c}=q_{i} .
$$

By Theorem 7(i) there exists an element $f=f_{k+1}$ of $A\left(\Delta_{k+1}, \Phi\right)$ which does not belong to $A\left(\bar{\Delta}_{k+1}, \Psi_{k+1}\right)$; we can assume that $f_{k+1} \in X_{m_{k+1}}$ for some large $m_{k+1}$. We may further assume that

$$
m_{k+1}>2 k+2 ; \quad \delta_{m_{k+1}} \leqq \frac{\rho}{2^{k+1-i}} \delta_{m_{i}} \quad \text { for } i<k+1 .
$$


Then

$$
\begin{aligned}
& E_{n}^{\Phi}\left(f_{k+1}\right) \leqq \frac{\rho}{2^{k+1-i}} \delta_{n} \quad \text { for } m_{i} \leqq n<m_{i+1}, i \leqq k, \\
& E_{n}^{\Psi_{k+1}}\left(f_{k+1}\right)=0 \quad \text { for } n \geqq m_{i+1} .
\end{aligned}
$$

On the other hand,

$$
E_{n}^{\boldsymbol{\Psi}_{k+1}}\left(f_{k+1}\right)>\bar{\delta}_{n}^{(k+1)} \quad \text { for at least one } n .
$$

For $n<k+m_{k}, \bar{\delta}_{n}^{k+1}=\delta_{0}$ and since

$$
E_{n}^{\Psi_{k+1}}\left(f_{k+1}\right) \leqq\left\|f_{k+1}\right\|=E_{0}^{\Phi}\left(f_{k+1}\right) \leqq \delta_{0},
$$

we must have $n \geq k+m_{k}$ for our $n$. Selecting one such $n$ that satisfies (20), we put $n=k+n_{k}$. Increasing $m_{k+1}$, if necessary, we have $m_{k} \leq n_{k+1}<m_{k+1}$. For $n=n_{k+1}$,

$$
E_{n}^{\Psi}\left(f_{1}+\cdots+f_{k}+f_{k+1}\right) \geqq E_{n+k}^{\Psi_{k+1}}\left(f_{k+1}\right)>2 \rho \delta_{n} .
$$

We put $f=\sum_{k-1}^{\infty} f_{k}$. If $m_{i} \leq n<m_{i+1}$, then by (19),

$$
E_{n}^{\Phi}(f)=E_{n}^{\Phi}\left(\sum_{k=i+1}^{\infty} f_{k}\right) \leqq \sum_{k=i+1}^{\infty} E_{n}^{\Phi}\left(f_{k}\right) \leqq \sum_{k=i+1}^{\infty} \frac{\rho}{2^{k-i}} \delta_{n}=\rho \delta_{n}<\delta_{n} .
$$

This shows that $f \in A(\Delta, \Phi)$. On the other hand, for $n=n_{k}, k=1,2, \cdots$, $E_{n}^{\Psi}(f) \geqq E_{n}^{\Psi}\left(f_{1}+\cdots+f_{k}\right)-\sum_{i=k+1}^{\infty}\left\|f_{i}\right\| \geqq 2 \rho \delta_{n}-\sum_{i=k+1}^{\infty} \frac{\rho}{2^{i+1-k}} \delta_{n} \geqq \rho \delta_{n}$.

Theorem 7 (for a Hilbert space $X$ ) was given in Lorentz [23]. For special spaces $X\left(X=C\right.$ and $\left.X=L^{p}\right)$ and $\delta_{n}=n^{-\alpha}, \alpha>0$, Theorem 9 appears in Lorentz [21]. Olevskiir [27] has an interesting counterpart of this when $X=C[a, b], A=\operatorname{Lip} \alpha, 0<\alpha<1$ : For each system $\Phi$ there exists a function $f \in \operatorname{Lip} \alpha$, with the property that $E_{n}^{\Phi}(f)$ $\geqq C \omega(f, 1 / n), C>0$ for all $n=1,2, \cdots$. Here $\omega(f, 1 / n)$ is, possibly, much smaller than $n^{-\alpha}$, which serves as $\delta_{n}$.

\section{Review of recent results on entropy.}

5.1. Computation of entropy.

5.1.1. Continuous and differentiable functions. First we introduce some notations (compare [24, Chapter 3]). Let $r=0,1, \cdots$ be an integer, $\omega$ a modulus of continuity, $S$ an $s$-dimensional parallelepiped, 
$a_{i} \leqq x_{i} \leqq b_{i}, i=1, \cdots, s$. Let $\Lambda_{r \omega}^{s}=\Lambda_{r \omega}^{s}\left(S ; M_{0}, \cdots, M_{r}\right)$ be the class of all functions $f\left(x_{1}, \cdots, x_{s}\right)$ on $S$ which have all partial derivatives $\left|D^{(k)} f\right| \leqq M_{k}, k=0, \cdots, r$. In addition, the modulus of continuity of each $r$ th derivative should not exceed $\omega(h)$. We write $\Lambda_{\omega}$ for the class $\Lambda_{0 \omega}, \operatorname{Lip} \alpha$ for $\Lambda_{\omega}, \omega(h)=M h^{\alpha}$. In case $s=0$, functions $f$ may be defined on an arbitrary compact metric space $B$. The theorems about the degree of approximation of the classes $\Lambda_{r \omega}^{s}(S)$ by algebraic polynomials are well known (see [35, pp. 279 and 363], [24, Chapters 4, $5,6])$. They imply that $\Lambda_{r w}^{s}$ is contained between two full approximation sets of the space $C[S]$ of continuous functions: $A\left(\Delta^{\prime}\right) \subset \Lambda_{r \omega}^{s}$ $C A(\Delta)$, where both $\delta_{n}$ and $\delta_{n}^{\prime}$ are of the form $C n^{-r / 8} \omega\left(C^{\prime} n^{-1 / 8}\right)$. An application of Theorem 2 leads to the result:

$$
\frac{C_{1}}{\delta(\beta \epsilon)^{s}} \leqq H_{e}\left(\Lambda_{r \omega}^{*}\right) \leqq \frac{C_{2}}{\delta(\gamma \epsilon)^{s}},
$$

where $C_{1}, C_{2}, \beta, \gamma$ are positive constants, and $\delta=\delta(\epsilon)$ is defined by the equation $\delta^{r} \omega(\delta)=\epsilon$. If $r \geqq 1$, or if $r=0, \omega(h)=h^{\alpha}, 0<\alpha \leqq 1$, (1) leads to an asymptotic determination of $H_{\mathrm{e}}(A)$, namely

$$
H_{\epsilon}(A) \approx \frac{1}{\delta(\epsilon)^{s}} .
$$

With a different proof, results (1) and (2) are due to Kolmogorov (in [19], they are proved for $\left.\omega(h)=h^{\alpha}\right)$. For moduli of continuity $\omega(h)$ which increase more rapidly than $h^{\alpha}$ at $h=0$, one does not get in this way precise formulas for the entropy. According to Timan [36], however, (2) still holds for each concave modulus of continuity, in the corrected form

$$
H_{\epsilon}\left(\Lambda_{\omega}^{s}\right) \approx\left\{\frac{1}{\omega^{-1}(2 \epsilon)}\right\}^{s}
$$

This is obtained by finding an $M=M(\epsilon)>0$ for which the class $\Lambda_{\omega}^{s}(B)$ is approximated by $\Lambda_{w}^{s}(B), w(h)=M h$ with an error $\leqq \frac{1}{2}[\omega(\epsilon)-M \epsilon]$ (compare [24, p. 122]). It remains to take advantage of the very precise formulas for the entropy, that are valid for the class $\Lambda_{w}^{s}([19$, pp. 14, 78]).

Vosburg [40] improves a little the inequalities (1) if $\Lambda=\operatorname{Lip}(\alpha, B)$ and if $B$ is an arbitrary compact set contained in $[-1,+1]$. In this way he shows: $H_{\epsilon}(\operatorname{Lip}(\alpha, B))=o\left(\epsilon^{-1 / \alpha}\right)$ if and only if the set $B$ has measure zero.

The set of continuous functions is not compact in the uniform 
norm. However, the set $G$ of all graphs of continuous functions in the unit square is compact in the Hausdorff metric space of closed sets in the plane. Its entropy is $H_{\epsilon}(G) \approx(1 / \epsilon) \log (1 / \epsilon)$ (Clements [6], Penkov and Sendov [29]). Another result of Clements [6] concerns the class $V_{\alpha}$ of functions of bounded variation on $[0,1]$, which belong to $\operatorname{Lip} \alpha$, in the uniform norm. Then $H_{\epsilon}\left(V_{\alpha}\right) \approx(1 / \epsilon) \log (1 / \epsilon)$ for each $\alpha, 0<\alpha<1$. An interesting unsolved question is the determination of the widths of the sets $V_{\alpha}$. Brudnyi and Kotljar [4], [20] study the entropy of classes $A$ of functions $f\left(x_{1}, \cdots, x_{s}\right)$ that have different properties with respect to each variable $x_{i}$. They assume that for each $i$, the $k_{i}$ th difference of $f$ with respect to $x_{i}$ satisfies

$$
\left|\Delta_{h}^{k_{i}} f\left(x_{1}, \cdots, x_{s}\right)\right| \leqq M_{i} h^{\beta_{i}},
$$

where $0<\beta_{i} \leqq k_{i}$, and derive, for this class $A, H_{\epsilon}(A) \approx(1 / \epsilon) \Sigma_{1}^{n} \beta_{i}^{-1}$. This also follows from our Theorem 2 and results about polynomial approximation (Timan [35, p. 279]).

5.1.2. The $L^{p}$-norm. Analogues of classes $\Lambda$ of 5.1 .1 can be defined in the $L^{p}$-norm. They are denoted $\Lambda_{r \omega}^{s p}(S)$. The known approximation theorems [35] and Theorem 2 lead to statements of the type (2). For example we have:

Theorem 10. The set $A=\operatorname{Lip}(\alpha, p), 0<\alpha \leqq 1, p \geqq 1$ of functions $f$ on $[0,1]$ for which $\int_{0}^{1}|f|^{p} d x \leqq 1$ and $\int_{0}^{+1}|f(x+h)-f(x)|{ }^{p} d x \leqq C h^{p \alpha}$ has the entropy $H_{\epsilon}(A) \approx(1 / \epsilon)^{s / \alpha}$. Similarly,

$$
H_{\epsilon}\left(\Lambda_{r \alpha}^{s p}\right) \approx(1 / \epsilon)^{s /(r+\alpha)} .
$$

These results seem to be new. For similar classes in $L^{2}$, for which our Theorem 2 can also be used, Brudnyì and Timan [5] obtain as an upper estimate only $(1 / \epsilon)^{s /(r+\alpha)} \log (1 / \epsilon)$.

Golovkin [13], for certain classes $A$, similar to $\Lambda_{r \alpha}^{s p}$, in spaces of functions with monotone and translation-invariant norm $(\|f\|$ is monotone if it increases whenever $|f|$ increases), announces estimates of $H_{\epsilon}(A)$ from above and below. They are of great generality, but not very precise. In many cases he determines the limit

$$
\lim _{\epsilon \rightarrow 0}\left\{\log H_{\epsilon}(A) / \log \frac{1}{\epsilon}\right\} \text {. }
$$

Smoljak [32] considers sets of functions on the $s$-dimensional torus,

$$
f\left(x_{1}, \cdots, x_{s}\right)=\sum_{m_{1}, \ldots, m_{s}=-\infty}^{+\infty} c_{m_{1} \ldots m_{s}} \exp i\left(m_{1} x_{1}+\cdots+m_{s} x_{s}\right),
$$


in the $L^{2}$-metric, that are restricted by some asymptotic properties of their Fourier coefficients. For the class $E_{s}^{\alpha k}(M)$ this restriction is

$$
\left|c_{m_{1} \ldots m_{s}}\right| \leqq M\left(\bar{m}_{1} \cdots \bar{m}_{s}\right)^{-\alpha}\left[\log ^{k}\left(\bar{m}_{1} \cdots \bar{m}_{s}\right)+1\right] \text {, }
$$

where $\bar{m}=|m|$ if $m \neq 0,0=1, \alpha>\frac{1}{2}, k \geqq 0$, and for $W_{s}^{\alpha}(M)$,

$$
\sum\left|\left(\bar{m}_{1} \cdots \bar{m}_{s}\right)^{\alpha} c_{m_{1} \ldots m_{s}}\right|^{2} \leqq M^{2}
$$

He finds:

$$
\begin{aligned}
& H_{\epsilon}\left(E_{s}^{\alpha k}\right) \approx\left(\frac{1}{\epsilon}\right)^{1 /(\alpha-1 / 2)} \log (2 k+2 \alpha(s-1)) /(2 \alpha-1) \frac{1}{\epsilon}, \\
& H_{\epsilon}\left(W_{s}^{\alpha}\right) \approx\left(\frac{1}{\epsilon}\right)^{1 / \alpha} \log ^{s-1} \frac{1}{\epsilon} .
\end{aligned}
$$

Arranging the functions $\phi=\exp i\left(k_{1} x_{1}+\cdots+k_{8} x_{s}\right)$ in proper order, we can compute from (6) and (7) upper bounds for the degrees of approximation $\delta_{n}$, and imbed the above classes into sets $A(\Delta, \Phi)$. In this way, the upper (harder) estimates for the entropies given by (8) follow from our Theorem 2 .

An interesting problem is to derive, from the behavior of $H_{\mathrm{e}}(A)$, properties of the Fourier coefficients of some, or of most functions $f \in A, A \subset L^{p}$ or $A \subset C$. To give only one example: Is it true, for a subset $A$ of $C$, that $H_{\epsilon}(A) \geqq$ Const. $(1 / \epsilon)^{2}$ implies the existence of a function $f \in A$ whose Fourier series is not absolutely convergent?

5.1.3. Analytic functions. For analytic functions, excellent approximation theorems are known. They can be used for the computation of entropies. This applies to Vituškin's estimates of classes $A$ of functions defined on polycylinders [37, Chapter 2], [24, pp. 156-157]. Each of these classes is contained between two full approximation sets. The upper class is obtained from the approximation of functions $f \in A$ by partial sums of their Taylor series; the lower class is derived from inverse theorems of Bernšter̆n's type. The term $S_{j}$ of 3(11) gives the remainder of Vituṣ̌kin's formula. Erohin [12] proves the formula

$$
H_{\epsilon}(A(K, G ; M)) \sim \frac{1}{\log R} \log ^{2}\left(\frac{1}{\epsilon}\right),
$$

where $A=A(K, G ; M)$ is the set of functions, analytic in the open set $G$, with $|f(z)| \leqq M, z \in G$, in the uniform norm on the compact set $K \subset G ; R$ is the conformal radius of the pair $K, G$. This follows at 
once from our Theorem 2 and Theorem 3 in Erohin [11], which deals with approximation of functions $f \in A$ by linear combinations of elements of a properly chosen basis $\Phi$. [The last quoted theorem should probably contain two different constants, for a lower and an upper estimate, instead of a single one $C(\delta)$.]

For some classes of functions analytic in a strip and for classes of harmonic functions, Tihomirov [34] has a better remainder, namely $O(\log (1 / \epsilon))$, instead of Vituškin's $O(\log (1 / \epsilon) \log \log (1 / \epsilon))$. The new method of proof is based on some geometric properties of sets of Fourier coefficients.

$\mathrm{Al}^{\prime}$ per [2] studies classes $T_{q}$ of functions $f$ analytic in a region $G$, bounded by a curve $\Gamma$ and continuous on $\bar{G}$; the functions satisfy the conditions $\left|f^{(k)}(z)\right| \leqq C_{k}, k=0, \cdots, r, f^{(r)} \in \operatorname{Lip} \alpha$ on $\bar{G} ; q=r+\alpha$, $0<\alpha \leqq 1$. Al'per refers to his own approximation theorems of functions $f \in T_{q}$; at present, more powerful results of Dzjadyk [9, Theorem 4.1]; [10, Theorem 1.7] are known, which allow even angular points of $\Gamma$. One obtains in this way $H_{\epsilon}\left(T_{q}\right) \approx(1 / \epsilon)^{1 / q}$, if $\Gamma$ is smooth except for angles; $\mathrm{Al}^{\prime}$ per also has $\log H_{\epsilon}\left(T_{q}\right) \sim(1 / q) \log (1 / \epsilon)$, if $\Gamma$ has a continuously turning tangent, and $\log H_{\epsilon}\left(T_{q}\right) \approx \log (1 / \epsilon)$, if $\Gamma$ is a rectifiable Jordan curve.

5.1.4. Compact sets in Banach spaces. Compact sets in Hilbert and Banach spaces were treated by Brudnyǐ and Timan [5], see also [35]. Most of their results are special cases of ours. (Compare \$3, §4.) HelemskiI and Henkin [14] use entropy in order to characterize the size of ellipsoids $D$ which contain a given compact subset $A$ of a Hilbert space. One of their results is that if $\lim \sup \left\{H_{\epsilon}(A) / \log (1 / \epsilon)\right\}$ $<q<2$, then there exists an ellipsoid $D \supset A$ with

$$
\limsup _{\epsilon \rightarrow 0}\left\{H_{\epsilon}(D) / \log \frac{1}{\epsilon}\right\}<\frac{2 q}{2-q} .
$$

It should be noted, that for ellipsoids $D$ in spaces $l^{p}, 1 \leqq p \leqq+\infty$, Mitjagin [25, Theorem 3] obtained inequalities similar to or even better than the inequalities of our Theorem 2. An ellipsoid in $l^{p}$ is the set of all points $x=\left(x_{1}, x_{2}, \cdots\right)$ for which $\sum_{n=1}^{\infty}\left|x_{n} / \delta_{n}\right|{ }^{p} \leqq 1$, where $\delta_{n}>0, \delta_{n} \rightarrow 0$ is a given decreasing sequence. For arbitrary compacts he gives inequalities that connect $H_{\epsilon}(A)$ and the widths $d_{n}(A)$ [25, Theorem 4], [24, p. 164]. They do not imply 3(5).

5.2. Applications of entropy.

5.2.1. Invariants of linear topological spaces. Entropy-theoretic notions can be used in order to construct invariants of linear topological spaces. The approximate dimension of a linear topological space, de- 
fined by Kolmogorov [18] is less fine but easier to handle than Banach's dimension. All infinite-dimensional Banach spaces have the same (maximal) approximate dimension. This means that the new notion is fruitful only for topological spaces that are in some sense close to finite dimensional spaces, for example, for spaces of analytic functions. Pelczyński [28] also defined an invariant of this kind. Computing the approximate dimension, Kolmogorov showed that the spaces of analytic functions of different number of variables are not linearily homeomorphic. Similarly, Pelczyński [28] proved that the spaces of entire functions of one variable and of functions analytic in $|z|<1$ are topologically different. Compare the article of Rolewicz [30].

Another interesting application of entropic notions is a characterization of nuclear spaces among all spaces of type $F$, by Gel'fand and Mitjagin [25].

5.2.2. Superpositions of functions. According to Kolmogorov ([17], slightly improved in [24, Chapter 11]) there exist fixed continuous functions $\phi_{i}, \psi_{i}, i=1, \cdots, 5$, that map $[0,1]$ into itself and have the property that each function $f(x, y)$, continuous on the square $0 \leqq x$, $y \leqq 1$, is representable in the form

$$
f(x, y)=\sum_{i=1}^{5} g\left(\phi_{i}(x)+\psi_{i}(y)\right)
$$

Here $g(u), 0 \leqq u \leqq 2$ is some continuous function, depending on $f$. One can assume that the functions $\phi_{i}, \psi_{i}$ belong to the class Lip $\alpha$ for each $0<\alpha<1$. There exists a conjecture of Kolmogorov, ${ }^{2}$ which, in its general form, states the following: Not all analytic functions of two variables are representable by means of superpositions of continuously differentiable functions of one variable and of addition; not all analytic functions of three variables are representable by superpositions of continuously differentiable functions of two variables. For "linear superpositions," such as (10), this has been proved by Vituškin [38, 39]. One of Henkin's [15] improvements of his results is the following interesting theorem. Assume that the functions $p_{i}, \phi_{i}$, $i=1, \cdots, N$ are continuous in the whole plane, and that the $\phi_{i}$ are continuously differentiable. Then for each region $D$ in the plane there are natural numbers $n, m$ for which the polynomial $(x+n y)^{m}$ is not equal to any superposition

\footnotetext{
${ }^{2}$ Communication of D. Sprecher.
} 


$$
\sum_{i=1}^{N} p_{i}(x, y) g_{i}\left(\phi_{i}(x, y)\right)
$$

where $g_{i}$ are arbitrary bounded measurable functions. The proof depends upon the comparison of entropies $H_{\epsilon}^{\delta}(F)$ of sets $F$ of functions $f(x, y)$ of the form (11) and of analytic functions, in the metric

$$
\|f\|=\sup _{U_{\delta} \subset D}\left|\frac{1}{\pi \delta^{2}} \int_{-U_{\delta}} \int f(u, v) d u d v\right|,
$$

where $\delta>0$ is a properly chosen function of $\epsilon$, and $U_{\delta}$ is an arbitrary circle of radius $\delta$.

As a corollary, representation (10) is not always possible if $\phi_{i}, \psi_{i}$ are continuously differentiable.

6. Vituškin's theorems in the uniform metric. The last two sections of this paper are devoted to the exposition of results from Vituškin's theory of nonlinear approximation, developed by him in his book [37]. In this section, we deal with sets of continuous and differentiable functions in the uniform metric. The main advantage of our approach in this section is its simplicity: we avoid the use of multidimensional variations [37, Chapter 4]. The simple combinatorial device which we use appears also in H. S. Shapiro [31]; see Remark at the end of $\$ 6.2$. As Vituškin does, we use in an essential way Oleinnik's estimates [26]; [37, p. 132]. In this way we obtain Theorem 13 below, which is essentially identical with Theorem 3 [37, p. 186] of Vituškin about piecewise rational approximation with moving barrier. This method is such that each improvement of Oleinik's estimate would automatically lead to some improvement of the final inequalities. For the case of a constant barrier and for "rational continuous" approximation, Vituškin has slightly better results (Theorem 12 below), which do not seem to follow by our method.

In $\$ 7$ we will show that theorems of Vituškin's type are not restricted to uniform metric. Results obtained there are valid for polynomial approximation in Banach and $L^{p}$ spaces. Here again we use the method of $\$ 3$ for the computation of entropies.

6.1. Definitions and lemmas. Let $P(t)$ be a polynomial in $t=\left(t_{1}, \cdots, t_{n}\right)$, of degree $p$ in the variables $t_{i}$ jointly, which is not a constant. The equation $P(t)=0$ splits the space $R^{n}$ into a collection of connected sets. Important for us is an upper bound for their number, contained in the following lemma. 
Lemma 7 (Oleìnik). The equation $P(t)=0$ defines at most $2 p^{n}$ bounded components of $R^{n}$, and at most

$$
2(p+2)^{n}
$$

connected bounded or unbounded components ( $p \geqq 2, n \geqq 1$ ).

According to Oleynik [26], the number of bounded components of $R^{n}$ defined by $P(t)=0$ is at most $(p-1)^{n}+2 n$ if $p$ is odd, and at most

$$
\frac{1}{2} \frac{(p-1)^{n+1}-(p-1)}{p-2}+\frac{n(n+1)}{4}
$$

if $p$ is even. Each of these numbers does not exceed $2 p^{n}$ if $p \geqq 2, n \geqq 1$. The second statement of the lemma follows from the fact that the number of components of $R^{n}$, defined by $P(t)=0$, which intersect the ball $t_{1}^{2}+\cdots+t_{n}^{2} \leqq a^{2}$ does not exceed the number of bounded components of $R^{n}$, defined by the equation $P(t)\left(t_{1}^{2}+\cdots+t_{n}^{2}-a^{2}\right)=0$.

In the classical theory of linear approximation, functions $f(x)$, $x \in B$, which belong to a given class, are approximated by linear expressions

$$
t_{1} \phi_{1}(x)+\cdots+t_{n} \phi_{n}(x),
$$

where the functions $\phi_{i}(x), x \in B$ are given, and $t=\left(t_{1}, \cdots, t_{n}\right) \in R^{n}$ can be selected for a given $f$. With Vituškin [37], we consider more general expressions. A rational expression of degree $p$ is a quotient

$$
R(x, t)=\frac{P(x, t)}{Q(x, t)}, \quad x \in B, t \in R^{n},
$$

where $P$ and $Q$ are polynomials of degree $p$ in $t_{i}, i=1, \cdots, n$, with coefficients which are given functions of $x .^{3}$ There may exist some $x, t$ for which $R(x, t)$ is not defined, in other words, $Q(x, t)=0$. More general are piecewise rational expressions $R(x, t)$. Let

$$
\widetilde{P}(x, t)
$$

be a polynomial of degree $q$ in $t=\left(t_{1}, \cdots, t_{n}\right)$, with coefficients which are given functions of $x$. According to Lemma 7 , for each $x, \widetilde{P}(x, t)=0$ decomposes $R^{n}$ into at most $2(q+2)^{n}$ sets $\Gamma_{j}=\Gamma_{j}(x)$. On the closure of each $\Gamma_{j}$ we put

$$
R(x, t)=\frac{P_{j}(x, t)}{Q_{j}(x, t)},
$$

${ }^{3}$ Vituškin [37] formulates his theorems for the case when $P, Q$ are polynomials of degree $p$ in each $t_{i}, i=1, \cdots, n$ separately. Since his proofs are based on Lemma 7 , this seems to be unjustified. 
where $P_{j}, Q_{j}$ are polynomials of degree $p$ in $t$. This $R(x, t)$ is called a piecewise rational expression of degree $p$ with barrier (3) of degree $q$. One of the justifications for the study of piecewise rational approximations lies in the fact that operations of taking maxima or minima, performed on rational expressions lead to piecewise rational expressions.

Sometimes simpler results are valid for piecewise rational approximations $R(x, t)$ with barrier $\widetilde{P}(t)=0$ independent of $x$.

If $Q(x, t) \equiv 1$ in (2), we obtain a polynomial expression of degree $p$, or a piecewise polynomial expression of degree $p$ with barrier of degree $q$. This case lends itself to generalizations for arbitrary Banach spaces.

We shall say that $R(x, t)$ is an $\epsilon$-approximation of a class $A$ of functions $f \in C(B)$, if for each $f \in A$ there is a $t \in R^{n}$ for which $R(x, t)$ is defined for all $x \in B$ and satisfies

$$
|f(x)-R(x, t)|<\epsilon, \quad x \in B .
$$

If $A$ is a subset of a Banach space $X$, and $P(t)$ is a polynomial (or piecewise polynomial) expression whose coefficients are elements of $X$, we can say that $P$ provides an $\epsilon$-approximation for $A$ if for each $f \in A$ there is a $t \in R^{n}$ with the property that

$$
\|f-P(t)\|<\epsilon .
$$

6.2. Approximation of sets $\Lambda$ in the uniform norm. For an $\epsilon$-approximation $R(x, t)$ of a set $A \subset C(B)$ we would like to obtain a lower bound for the numbers $n, p, q$.

Let $A$ be a set of continuous functions on a set $B$, let $\epsilon>0$, and assume that $B$ contains $M=M(\epsilon)$ points $x_{1}, \cdots, x_{M}$ such that for each distribution of signs $\lambda=\left(\lambda_{\mu}\right), \mu=1, \cdots, M, \lambda_{\mu}= \pm 1$, there is a function $f \in A$ for which

$$
\left|f\left(x_{\mu}\right)\right| \geqq \epsilon, \quad \operatorname{sign} f\left(x_{\mu}\right)=\lambda_{\mu}, \quad \mu=1, \cdots, M .
$$

TheOREM 11. If a set $A$ of the above kind has an e-approximation $R(x, t), t=\left(t_{1}, \cdots, t_{n}\right)$, which is piecewise rational of degree $p$ with barrier of degree $q$, then

$$
n \log (p+q+M) \geqq C M,
$$

where $C$ is an absolute constant.

Proof. For each of the $2^{M}$ distributions of signs $\lambda$, let $f_{\lambda} \in A$ be the function $f$ which satisfies (7). Let $t^{\lambda} \in R^{n}$ be the value of $t$ which satisfies (5). Then $\left|f_{\lambda}\left(x_{\mu}\right)-R\left(x_{\mu}, t^{\lambda}\right)\right|<\epsilon$ shows that all values $R\left(x_{\mu}, t^{\lambda}\right)$ are different from zero and of the same sign as the $f_{\lambda}\left(x_{\mu}\right)$. It follows that for each pair $\lambda \neq \lambda^{\prime}$, there is a $\mu$ for which $R\left(x_{\mu}, t^{\lambda}\right)$ and $R\left(x_{\mu}, t^{\lambda^{\prime}}\right)$ 
are of different sign.

We consider the equation of degree $M q$,

$$
\prod_{\mu=1}^{M} \widetilde{P}\left(x_{\mu}, t\right)=0,
$$

which, by Lemma 7 , decomposes $R^{n}$ into $N \leqq 2(M q+2)^{n}$ connected sets $\Gamma_{j}, j \leqq N$. On each $\Gamma_{j}$, each $R\left(x_{\mu}, t\right)$ is the quotient of two polynomials,

$$
R\left(x_{\mu}, t\right)=P_{j \mu}(t) / Q_{j \mu}(t), \quad \mu=1, \cdots, M, \quad t \in \Gamma_{j} .
$$

For a fixed $j$, the equation

$$
\prod_{\mu=1}^{M} P_{j \mu}(t) Q_{j \mu}(t)=0
$$

decomposes $R^{n}$ into at most $2(2 M p+2)^{n}$ components, on the interior of each of which the $R\left(x_{\mu}, t\right)$ do not vanish; on their closures the $R\left(x_{\mu}, t\right)$ keep a constant sign. Intersecting these closures with the set $\Gamma_{j}$, we obtain sets $\Delta_{v j}$, at most $4(M q+2)^{n}(2 M p+2)^{n}$ in number, with the following property: if $t, t^{\prime} \in \Delta_{v j}$, then $R\left(x_{\mu}, t\right)$ and $R\left(x_{\mu}, t^{\prime}\right)$ are of the same sign for all $\mu$.

It follows that the number of points $t^{\lambda}$ cannot exceed the number of the sets $\Delta_{p j}$, or that

$$
2^{M} \leqq 4(M q+2)^{n}(2 M p+2)^{n} .
$$

This implies (8). Our Theorem 11 is essentially equivalent to Vituškin's Theorem 3, p. 186 in [37]. For certain representations, however, Vituškin has a better result. He calls a piecewise rational function $R(x, t)$ continuous if the denominator $Q_{j}(x, t)$ of the representation $R(x, t)=P_{j}(x, t) / Q_{j}(x, t), t \in \Gamma_{j}$, does not vanish for $x \in B$, $t \in \Gamma_{j}$. On the other hand, we have assumed in Theorem 11 only that $Q_{j}(x, t) \neq 0$ for $x \in B$ and for $t$ actually used in (5).

Theorem 12 (Vituškin [37, Theorem 1, p. 182]). If $R(x, t)$ is a continuous piecewise rational $\epsilon$-approximation of the set $A$ with barrier independent of $x$, then

$$
n \log (p+q+1) \geqq C_{1} M,
$$

where $C_{1}$ is an absolute constant.

We return to some applications of Theorem 11 . Let $A=\Lambda_{\omega}(B, c)$ be the class of continuous functions defined in $\$ 5$. Then, if $\Lambda$ has an $\epsilon$-approximation $R(x, t)$, 


$$
n \log (p+q+M) \geqq \text { Const. } H_{4 \epsilon}(\Lambda) \text {. }
$$

To prove this, we note that we can take $M$ equal to the maximal number $M_{\delta}, \delta=\omega^{-1}(2 \epsilon)$ of points of $B$ at distances $\geqq \delta$ from each other. By $[19$, p. $77,(234)]$,

$$
M_{\frac{1}{2} \omega^{-1}\left(\frac{1}{2} \epsilon\right)} \geqq H_{\epsilon}(\Lambda)-\log \frac{C}{\epsilon} .
$$

Therefore

$$
M=M_{\delta} \geqq H_{4 \epsilon}(\Lambda)-\log \frac{C}{\epsilon} \geqq \frac{1}{3} H_{4 \epsilon}(\Lambda),
$$

as $H_{\epsilon}(\Lambda) \geqq 2 \log (1 / \epsilon)$, since $B$ has at least two points. Thus (12) follows from Theorem 11 .

If we assume that moreover

$$
H_{\epsilon}(\Lambda) \leqq C \epsilon^{-C_{1}} \text {, }
$$

then from the inequality $[19$, p. $77,(235)]$ we have

$$
M_{2 \omega^{-1}(2 \epsilon)} \leqq \frac{1}{\log 2} H_{\epsilon}(\Lambda)
$$

and using the properties of a modulus of continuity and (13),

$$
M=M_{\omega^{-1}(2 \epsilon)} \leqq M_{2 \omega^{-1}(\epsilon)} \leqq \text { Const. } \epsilon^{-C_{1}},
$$

so that (12) implies

$$
n \log \left(p+q+\frac{1}{\epsilon}\right) \geqq \text { Const. } H_{4 \epsilon}(\Lambda),
$$

which is equivalent to Theorem $2[37$, p. 195] of Vituškin.

Similarly, let $\Lambda=\Lambda_{r, \omega}^{s}(S ; c)$, where $r \geqq 1$ and $S$ is an $s$-dimensional parallelepiped. Then (Vituškin [37, Theorem 2, p. 191]),

$$
n \log \left(p+q+\frac{1}{\epsilon}\right) \geqq \text { Const. } H_{\epsilon}(\Lambda) \text {. }
$$

It follows from [24, p. 136] that one can take $M \approx \delta^{-8}$, if $\delta$ is defined by $\delta^{r} \omega(\delta)=\epsilon$. Also, it is known that for some constants $K, L, \beta, \gamma$ $[24$, p. 153],

$$
K \delta(\beta \epsilon)^{-s} \leqq H_{\epsilon}(\Lambda) \leqq L \delta(\gamma \epsilon)^{-8} .
$$

It follows from this and the properties of the modulus of continuity that $H_{\epsilon}(\Lambda) \approx \delta(\epsilon)^{-8} \approx M$, so that (15) is a consequence of Theorem 11 . 
REMARK. Let $P(t), t=\left(t_{1}, \cdots, t_{n}\right)$ be a product of $p$ linear forms in $t$. In this case, Shapiro [31] found a better upper bound than that given in Lemma 7 for the number $N$ of components of $R^{n}$. He showed that $N \leqq p^{n} /(n-1)$ ! if $n \leqq p$. This has the following consequence. Let $R(x, t)=P(x, t) / Q(x, t)$ be a rational $\epsilon$-approximation of $\Lambda$ of degree 1 without barriers. Then

$$
n \geqq C M, \quad C>0 .
$$

For the proof consider the product of $2 M$ linear factors

$$
P(t)=\prod_{\mu=1}^{M} P\left(x_{\mu}, t\right) Q\left(x_{\mu}, t\right) .
$$

We can assume that $n<2 M$. Then we can use the estimate given above, and obtain, as in the proof of Theorem 11,

$$
(2 M)^{n} /(n-1) ! \geqq 2^{M} \text {. }
$$

This, after a simple computation, gives (16).

It should be noted, that Shapiro's inequality (16) does not follow from Vituškin's Theorem 12 , for $p=1, q=0$, since Vituškin assumes that the denominators $Q(x, t)$ do not vanish.

7. Vituškin's theorems for Banach spaces. Results similar to Theorem 11 hold also for sets $A$ in Banach spaces $X$. Of course, we must now replace rational expressions by polynomial expressions, since division, in general, has no meaning in $X$. The method of $\$ 7.1$ is close to that of $\S 6$ and is valid in $L^{p}$-spaces. The method of $\$ 7.2$ is somewhat different. It applies to sets $A(\Delta)$ in arbitrary spaces $X$, if the $\delta_{n}$ tend to zero sufficiently rapidly.

7.1. Approximation in $L^{p}$-norm. Let $A$ be a subset of the space $L^{p}$ of functions $f(x)$ defined on an $s$-dimensional parallelepiped $S$. Let $\widetilde{P}(t)$ be a polynomial of degree $q$ in $t=\left(t_{1}, \cdots, t_{n}\right)$, then $\widetilde{P}(t)=0$ decomposes the space $R^{n}$ into $N \leqq 2(q+2)^{n}$ connected sets $\Gamma_{j}$. For $x \in B$, $t \in \Gamma_{j}$ let $R(x, t)=P_{j}(x, t)$ be a polynomial in $t$ of degree $p$. The piecewise polynomial function $R(x, t)$ is an $\epsilon$-approximation of $A$ if for each $f \in A$ there is a $t \in R^{n}$ with the property

$$
\int_{S}|f(x)-R(x, t)|^{p} d x<\epsilon^{p}
$$

We shall use a method which appears in [21].

LEMMA 8. Let $Q$ be the set of all $2^{M}$ possible distributions of signs $\lambda=\left\{\lambda_{\mu}\right\}, \mu=1, \cdots, M, \lambda_{\mu}= \pm 1$, and $Q^{\prime}$ a subset of $Q$ which consists of not more than $\rho^{\mu}$ elements where $\rho<\frac{1}{3} 2^{5 / 3}$. Then for all large $M$, there 
is at least one distribution of signs $\lambda$ which is different from each $\lambda^{\prime} \in Q^{\prime}$ in at least $M / 3$ places.

Proof. Let $N=[M / 3]+1$. We count the number of all $\lambda$ which can be obtained from a given $\lambda^{\prime}$ by changes in at most $N$ places. This number $T$ does not exceed

$$
T \leqq 1+M+\frac{M(M-1)}{2}+\cdots+\left(\begin{array}{c}
M \\
N
\end{array}\right) \leqq(N+1) \frac{M !}{N !(M-N) !} .
$$

By Stirling's formula, the right-hand side is asymptotically $\sim M \log \left(3 / 2^{2 / 3}\right)$, so that $T<(2 / \rho)^{M}$ for large $M$. The number of $\lambda$ obtainable in this way from some $\lambda^{\prime} \in Q^{\prime}$, does not exceed $T \rho^{M}<2^{M}$, which completes the proof.

THEOREM 13. Let $\Lambda=\Lambda_{r \omega}^{s}(S, c), r \geqq 1$, be the subset of $C[S]$, and let $R(x, t)$, be a piecewise polynomial function of degree $p$ with barrier $\tilde{P}(t)=0$ of degree $q$, which is an $\epsilon$-approximation of $\Lambda$ in the $L^{1}$-norm on $S$. Then

$$
n \log \left(p+q+\frac{1}{\epsilon}\right) \geqq \text { Const. } H_{\epsilon}(\Lambda) .
$$

We assume here that $r \geqq 1$ or that $r=0$ and $\omega(t)=t^{\alpha}, 0<\alpha \leqq 1$; the entropy in (2) is in the uniform norm.

Proof. For each small $\delta>0, S$ contains $M \approx \delta^{-s}$ disjoint balls $U_{\mu}$ of radius $\delta$. Let $\delta$ be defined by $\delta^{r} \omega(\delta)=a \epsilon ; a>0$. Then we can find functions $f_{\lambda}, \lambda=\left\{\lambda_{\mu}\right\}, \lambda_{\mu}= \pm 1$ with

$$
\left|\int_{U_{\mu}} f d x\right| \geqq C \delta^{s} a \epsilon \geqq C_{1} \frac{a \epsilon}{M}, \underset{x \in U_{\mu}}{\operatorname{sign}} f(x)=\lambda_{\mu} .
$$

(See [24, p. 136].) We have

$$
\int_{S}|f(x)-R(x, t)| d x \geqq \sum_{\mu=1}^{M}\left|\int_{U_{\mu}} f d x-\int_{U_{\mu}} R(x, t) d x\right| .
$$

For $j=1, \cdots, N, \mu=1, \cdots, M$, we put

$$
\pi_{\mu j}(t)=\int_{U} P_{j}(x, t) d x=\int_{U_{\mu}} R(x, t) d x .
$$

The equation

$$
\pi_{j}(t)=\prod_{\mu=1}^{M} \pi_{\mu j}(t)=0
$$

decomposes $\Gamma_{j}$ into at most $2(M p+2)^{n}$ sets $\Delta_{l j}$, on each of which the 
$\pi_{\mu j}(t)$ are of constant sign. There are $T \leqq 4(M p+2)^{n}(q+2)^{n}$ sets $\Delta_{l j}$, and on each of them, each integral $\int_{U_{\mu}} R(x, t) d x$ is of constant sign. We show that $T>\rho^{M}$. Assume that $T \leqq \rho^{M}$, then by Lemma 8 , there is a distribution of signs $\lambda=\left\{\lambda_{\mu}\right\}$ which is different from any distribution of signs (5) in at least $\frac{1}{3} M$ places. Then for the corresponding $f_{\lambda}$, by (3) and (4),

$$
\int_{S}\left|f_{\lambda}(x)-R(x, t)\right| d x \geqq \frac{1}{3} M C_{1} \frac{a \epsilon}{M}>\epsilon, \text { for each } t,
$$

if $a>0$ is selected properly. Hence $T>\rho^{M}$, and we obtain

$$
n \log (M+p+q) \leqq \text { Const. } M \text {. }
$$

Because of the expressions for $\delta$ and $M$, and of the formula [24, p. 153] for the entropy of $\Lambda$, this is equivalent to (2).

The formulation of Theorem 12 was restricted to $p=1$ for the approximation and to $p=+\infty$ for the entropy because a similar statement for other values of the $p$ 's is a simple corollary.

Corollary. If $R$ is a piecerwise polynomial $\epsilon$-approximation of $\Lambda_{r \omega}^{p_{s}}(S, c)$ in the $L^{p_{1}-n o r m}$, then under the same conditions on $r$ and $\omega$ as in Theorem 13, we have relation (2), with $H_{\epsilon}(\Lambda)$ in $L^{p_{1}-n o r m}$.

For small $c_{1}>0, \quad \Lambda_{r \omega_{1}}^{s}\left(S, c_{1} c\right) \subset \Lambda_{r \omega}^{p s}(S, c)$, where $\omega_{1}=c_{1} \omega$; also, $\|f-P\|_{L^{p_{1}}<\epsilon \text { implies }}\|f-P\|_{L^{1}<c_{1}^{-1} \epsilon}$. Therefore our statement follows from Theorem 13 and the formula for $H_{\epsilon}\left(\Lambda_{r \omega}^{p s}\right)$ in $\$ 5$.

7.2. Classes $A(\Delta)$ with rapidly decreasing $\delta_{n}$. If $\delta_{n}$ decreases to zero rapidly, results better than those of $\$ 7.1 \mathrm{can}$ be obtained. For linear approximation, we could observe this phenomenon in $\$ 4$, compare $4(3)$ and 4(4). However, while 4(4) was true for the $\delta_{n}$ which satisfy $3(15)$ (this follows from Theorem 3 and $3(16)$ ), we have now to assume more (see condition (7) below). The results of this section should be compared with Vituškin's theorem [37, Theorem 1, p. 177] about analytic functions. The advantages of our results as compared to Vituškin's are the following: we treat arbitrary classes $A(\Delta, \Phi)$ (in arbitrary Banach spaces), which are described only in terms of the behavior of $\delta_{n}$, while Vituškin uses structural properties of the class $A$, in a space of continuous functions. He is able later to check these properties for classes of analytic functions. On the other hand, Vituškin can treat rational, instead of polynomial approximation.

Theorem 14. Let $P(t)$ be a polynomial $\epsilon$-approximation for $A(\Delta, \Phi)$ in $X$, of degree $p$ in $t=\left(t_{1}, \cdots, t_{n}\right)$. Assume that the numbers $\delta_{k}$ satisfy 


$$
\frac{\delta_{[n+\sigma n / \log n]}}{\delta_{n}} \rightarrow 0 \text { for each } \sigma>0
$$

Then

$$
n \log \left(p+\frac{1}{\epsilon}\right) \geqq(1-o(1)) H_{\epsilon}(A), \quad \epsilon \rightarrow 0 .
$$

Proof. Let the $N_{i}$ be defined by 3(1) with $C=e$. We note some consequences of the assumption (6). It follows from 3(2) that $\delta_{N_{i+1}} \geqq e^{-2} \delta_{N_{i}}$, hence (6) implies $N_{i+1} \leqq N_{i}+\sigma N_{i} / \log N_{i}$, for each $\sigma>0$, therefore

$$
\Delta N_{i} \log \Delta N_{i}=o\left(N_{i}\right), \quad i \rightarrow \infty .
$$

Next, condition (6) implies 3(15), and we derive from 3(20) that

$$
H_{a \epsilon}(A) / H_{\epsilon}(A) \rightarrow 1, \quad \epsilon \rightarrow 0, \text { for each } a>0 .
$$

Easy calculations show that (6) implies

$$
\delta_{n} n^{\alpha} \rightarrow 0 \text { for each } \alpha>0 \text {. }
$$

Let $\epsilon>0$ be given, we define $j$ by 3(3) and put $N=N_{j}-1$. Without changing the set $A(\Delta)$, we can replace the functions $\phi_{1}, \cdots, \phi_{N}$ by any linearly independent set of their linear combinations. Using also a standard result about finite dimensional Banach spaces (the lemma of Auerbach, see for example [35, p. 393]) we can assume the existence of linear functionals $L_{1}, \cdots, L_{N}$ on $X_{N}$, that together with the $\phi_{k}$ satisfy

$$
\begin{aligned}
\left\|L_{k}\right\| & =\left\|\phi_{k}\right\|=1, \\
L_{k}\left(\phi_{l}\right) & =0, k \neq l, L_{k}\left(\phi_{k}\right)=1, \quad k, l=1, \cdots, N .
\end{aligned}
$$

The set $B \subset X$ consists of all $g \in X$ of the form

$$
g=3 \epsilon \sum_{i=0}^{j-1} \sum_{N_{i} \leqq k<N_{i+1}} \lambda_{k} \phi_{k},
$$

where the $\lambda_{k}$ are integers which satisfy

$$
\left|\lambda_{k}\right| \leqq \frac{1}{3 \epsilon \Delta N_{i}} e^{-i-2}, \quad N_{i} \leqq k<N_{i+1}, \quad i=0, \cdots, j-1 .
$$

For $g$ of the form (12), $L_{k}(g)=3 \epsilon t_{k}$. We note some properties of the set $B$ :

(a) If $g, g^{\prime} \in B, g \neq g^{\prime}$, then $\left|L_{k}(g)-L_{k}\left(g^{\prime}\right)\right| \geqq 3 \epsilon$ for at least one $k$.

(b) We have $B \subset A(\Delta)$. This follows from the inequality 


$$
\begin{aligned}
\rho\left(g, X_{k}\right) & \leqq\left\|3 \epsilon \sum_{k<l \leqq N} \lambda_{l} \phi_{l}\right\| \leqq 3 \epsilon \sum_{i=i_{0}}^{j-1} \sum_{N_{i} \leqq l<N_{i}+1}\left|\lambda_{l}\right| \\
& \leqq \sum_{i=i_{0}}^{j-1} e^{-j-2} \leqq e^{-i_{0}-1} \leqq \delta_{k}, \quad k=0, \cdots, N,
\end{aligned}
$$

where $i_{0}$ is defined by $N_{i_{0}} \leqq k<N_{i_{0}+\mathbf{1}}$.

(c) The number $M$ of elements of $B$ satisfies

$$
\log M \geqq(1-o(1)) H_{\epsilon}(A(\Delta)), \quad \epsilon \rightarrow 0 .
$$

Since $\epsilon \geqq e^{-j+1}$, the number of integers $\lambda_{k}$ which satisfy (13) is $\geqq e^{j-i-3} /\left(3 \Delta N_{i}\right)$, and the number of all sets $\left(\lambda_{1}, \cdots, \lambda_{N}\right)$ is $\geqq \prod_{i=0}^{j-3}\left(e^{j-i-3} / 3 \Delta N_{i}\right)^{\Delta N_{i}}$, therefore

$$
\log M \geqq \sum_{i=1}^{j-3} \Delta N_{i}(j-i-3)-\sum_{i=1}^{j-3} \Delta N_{i}\left(\log 3 \Delta N_{i}\right) .
$$

The first sum is $=N_{1}+\cdots+N_{j-3} \sim H_{\epsilon}(A(\Delta))$ by $3(20)$; the second sum is $o(1) H_{\epsilon}(A(\Delta))$ by (8), 3(16) and $3(20)$.

Lemma 9. Let $\pi_{1}, \cdots, \pi_{N}$ be polynomials of degree $p$ in $t=\left(t_{1}, \cdots, t_{n}\right)$. Let $\epsilon>0$ and let $t^{\mu}, \mu=1, \cdots, M$ be points of $R^{n}$ with the properties that

$$
\begin{gathered}
\left|\pi_{k}\left(t^{\mu}\right)-\pi_{k}\left(t^{\prime}\right)\right|>\epsilon \text { for at least one } k \text { if } \mu \neq \mu^{\prime}: \\
\left|\pi_{k}\left(t^{\mu}\right)\right| \leqq M_{k}, \quad k=0, \cdots, M .
\end{gathered}
$$

Then

$$
2\left[p \sum_{k=1}^{N}\left(\frac{2 M_{k}}{\epsilon}+1\right)+2\right]^{n} \geqq M .
$$

Proof. We can assume that none of the polynomials $\pi_{k}$ is constant. Let $\pi(t)$ be defined by

$$
\pi(t)=\prod_{k=1}^{N} \prod_{|s| \leqq M_{k} / \epsilon}\left\{\pi_{k}(t)-s \epsilon\right\} .
$$

The degree of $\pi$ does not exceed $\sum_{k=1}^{N}\left(2 M_{k} / \epsilon+1\right) p$, hence, by Lemma 7 , the space $R^{n}$ can be decomposed into at most

$$
M_{0}=2\left[p \sum_{k=1}^{n}\left(2 M_{k} / \epsilon+1\right)+2\right]^{n}
$$

connected sets $\Gamma_{j}$, on each of which $\pi$ does not change sign. In the interior of each $\Gamma_{j}, \pi$ does not vanish, hence each $\pi_{k}$ satisfies there an inequality 


$$
s^{\prime} \epsilon \leqq \pi_{k}(t) \leqq\left(s^{\prime}+1\right) \epsilon
$$

The same inequality holds on the whole of $\Gamma_{j}$ by continuity. It follows that no two points $t^{\mu}, t^{\mu^{\prime}}, \mu \neq \mu^{\prime}$, can belong to the same $\Gamma_{j}$. Hence $M_{0} \geqq M$.

Proof of Theorem 14. We consider the set $B \subset A(\Delta)$ defined by (12) and (13). The number $M$ of points $g_{\mu} \in B$ satisfies (14).

Putting $\pi_{k}(t)=L_{k}(P(t)), k=1, \cdots, N$, we obtain polynomials of degree $p$ in $t=\left(t_{1}, \cdots, t_{n}\right)$. Since $P(t)$ is an $\epsilon$-approximation of $A(\Delta)$, for each $g_{\mu} \in B, \mu=1, \cdots, M$ there is a $t^{\mu} \in R^{n}$ with $\left\|g_{\mu}-P\left(t^{\mu}\right)\right\|$ $\leqq \epsilon$. Since $L_{k}\left(g_{\mu}\right)=3 \epsilon \lambda_{k}^{\mu}$, where $\lambda_{k}^{\mu}$ are the coordinates of $g_{\mu}$ in (12), we have

$$
\left|3 \epsilon \lambda_{k}^{\mu}-\pi_{k}\left(t^{\mu}\right)\right|<\epsilon, \quad k=1, \cdots, N, \quad \mu=1, \cdots, M .
$$

Let $\mu \neq \mu^{\prime}$. Since the $\lambda_{k}^{\mu}$ are integers, it follows from (19) that

$$
\left|\pi_{k}\left(t^{\mu}\right)-\pi_{k}\left(t^{\prime}\right)\right|>\epsilon
$$

must hold for at least one $k$. To estimate the $\pi_{k}\left(t^{\mu}\right)$, we note that (19) and (13) imply

$$
\left|\pi_{k}\left(t^{\mu}\right)\right| \leqq \epsilon+3 \epsilon\left|\lambda_{k}^{\mu}\right| \leqq \epsilon+e^{-i-2} / \Delta N_{i}, \quad \text { if } \quad N_{i} \leqq k<N_{i+1}
$$

If we denote the term on the right by $M_{k}$, then by $3(3), M_{k} / \epsilon \leqq$ $1+e^{j-i-3} / \Delta N_{i}$. Applying Lemma 3 and estimating the sum in (17) we obtain

$$
2\left(3 p N_{j}+p e^{j-2}+2\right)^{n} \geqq M .
$$

By (10), $N_{j} \delta_{N_{j}} \rightarrow 0$, hence $N_{j} e^{-j} \rightarrow 0$, and from (21) and (14) it is easy to derive (7).

REMARK. A similar theorem is valid for a piecewise polynomial $\epsilon$-approximation of $A(\Delta)$ with a barrier $\tilde{P}(t)=0$ of degree $q$. Then one has, instead of (7),

$$
n \log (p+q+1 / \epsilon) \geqq(1-o(1)) H_{\epsilon}(A) .
$$

We omit the proof, which is based on ideas used for the derivation of the last two theorems; the polynomials (5) are now to be replaced by $\pi_{k j}(t)=L_{k}\left(P_{j}(t)\right)$.

\section{BIBLIOGRAPHY}

1. N. I. Achiezer, Theory of approximation, F. Ungar, New York, 1956.

2. S. Ja. Al'per, On e-entropy of certain classes of functions, Dokl. Akad. Nauk SSSR 132 (1960), 977-979 = Soviet Math Dokl. 1 (1960), 667-669. 
3. T. Bonnesen and W. Fenchel, Theorie der konvexen Körper, Ergebnisse der Math., Bd. 3, no. 1, Springer, Berlin 1934.

4. Ju. A. Brudnyl and B. D. Kotljar, The order of growth of €entropy on certain compact classes of functions, Dokl. Akad. Nauk SSSR 148(1963), 1001-1004 = Soviet Math. Dokl. 4 (1963), 196-199.

5. Ju. A. Brudnyr and A. F. Timan, Constructive characteristics of compact sets in Banach spaces and €-entropy, Dokl. Akad. Nauk SSSR 126 (1959), 927-930.

6. G. F. Clements, Entropies of sets of functions of bounded variation, Canad. J. Math. 15 (1963), 422-432.

7. —_ Entropies of several sets of real valued functions, Pacific J. Math. 13 (1963), 1085-1095.

8. Ph. J. Davis, Interpolation and approximation, Blaisdell, New York, 1963.

9. V. K. Dzjadyk, On the problem of S. M. Nikol'skǐ, Izv. Akad. Nauk SSSR, Ser. Mat. 23 (1959), 697-736.

10. - - To the theory of approximation of analytic functions, Izv. Akad. Nauk SSSR, Ser. Mat. 27 (1963), 1135-1164; English transl., Amer. Math. Soc. Transl. (2) 53 (1966), 253-284.

11. V. D. Erohin, Conform mappings of rings and the fundamental basis of the space of functions that are analytic in the elementary neighborhood of an arbitrary continuum, Dokl. Akad. Nauk SSSR 120 (1958), 689-692.

12. - - The asymptotic behavior of $\epsilon$-entropy of analytic functions, Dokl. Akad. Nauk SSSR 120 (1958), 949-952.

13. K. K. Golovkin, The $\epsilon$-entropy of some compact sets of differentiable functions in spaces with a monotone norm, Dokl. Akad. Nauk SSSR 158 (1964), 261-263 = Soviet Math. Dokl. 5 (1964), 1209-1211.

14. A. Ja. Helemskiǔ and G. M. Henkin, Imbeddings of compacts into ellipsoids, Vestnik Moskov. Univ., Ser. 1, Mat. Meh, no. 2 (1963), 3-12.

15. G. M. Henkin, Linear superpositions of continuously differentiable functions, Dokl. Akad. Nauk SSSR 157 (1964), 288-290 = Soviet Math. Dokl. 5 (1964), 948-950.

16. A. N. Kolmogorov, Asymptotic characteristics of some completely bounded metric spaces, Dokl. Akad. Nauk SSSR 108 (1956), 585-589.

17. - On the representation of continuous functions of several variables, Dokl. Akad. Nauk SSSR 114 (1957), 953-956.

18. —_ Linear dimension of topological vector spaces, Dokl. Akad. Nauk SSSR 120 (1958), 239-241.

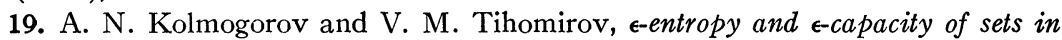
function spaces, Uspehi Mat. Nauk no. 2 (86), 14 (1959), 3-86; English transl., Amer. Math. Soc. Transl. (2) 17 (1961), 277-364.

20. B. D. Kotljar, The order of growth of e-entropy on classes of quasi-smooth functions, Uspehi Mat. Nauk no. 2 (110), 18 (1963), 135-138.

21. G. G. Lorentz, Lower bounds for the degree of approximation, Trans. Amer. Math. Soc. 97 (1960), 25-34.

22. —- Metric entropy, widths, and superpositions of functions, Amer. Math. Monthly 69 (1962), 469-485.

23. —-, Entropy and its applications, J. Soc. Indust. Appl. Math. Ser. B, Numer. Anal. 1 (1964), 97-103. 1966.

24. — Approximation of functions, Holt, Rinehart, Winston, New York 
25. B. S. Mitjagin, The approximative dimension and bases in nuclear spaces, Uspehi Mat. Nauk no. 4 (100), 16 (1961), 63-132.

26. O. A. Oley̌nik, Estimations of Betti numbers of real algebraic hypersurfaces, Mat. Sb. 28 (70) (1951), 635-640.

27. A. M. Olevskii, On a problem of P. L. Ul'janov, Uspehi Mat. Nauk no. 2 (122), 20 (1965), 197-202.

28. A. Pelczynski, On the approximation of $S$-spaces by finite dimensional spaces, Bull. Acad. Polon. Sci. Cl. III 5 (1957), 879-881.

29. B. Penkov and B1. Sendov, Entropy of the set of continuous functions of several variables, C. R. Acad. Bulgare Sci. 17 (1964), 335-337.

30. S. Rolewicz, On spaces of holomorphic functions, Studia Math. 21 (1962), 135160.

31. H. S. Shapiro, Some negative theorems of approximation theory, Michigan Math. J. 11 (1964), 211-217.

32. S. A. Smoljak, The e-entropy of classes $E_{\mathrm{a}}^{\alpha, k}(B)$ and $W_{\mathrm{a}}^{\alpha}(B)$ in the $L^{2}$-metric, Dokl. Akad. Nauk SSSR 131 (1960), 30-33 = Soviet Math. Dokl. 1 (1960), 192-195,

33. V. M. Tihomirov, Widths of sets in functional spaces and the theory of best approximations, Uspehi Mat. Nauk no. 3 (93), 15 (1960), 81-120.

34. V. M. Tihomirov, The $\epsilon$-entropy of some classes of periodic functions, Uspehi Mat. Nauk no. 6 (108), 17 (1962), 163-169.

35. A. F. Timan, Theory of approximation of functions of a real variable, Macmillan, New York, 1963.

36. - - The order of growth of $\epsilon$-entropy of spaces of real continuous functionals, defined on a connected compactum, Uspehi Mat. Nauk no. 1 (115), 19 (1964), 173-177.

37. A. G. Vituškin, Theory of transmission and processing of information, Pergamon Press, New York, 1961.

38. - Some properties of linear superpositions of smooth functions, Dokl. Akad. Nauk SSSR 156 (1964), 1003-1006 = Soviet Math Dokl. 5 (1964), 741-744.

39. - - Proof of existence of analytic functions of several variables, not representable by linear superpositions of continuously differentiable functions of fewer variables, Dokl. Akad. Nauk SSSR 156 (1964), 1258-1261 = Soviet Math. Dokl. 5 (1964), 793796.

40. A. Vosburg, Metric entropy of certain classes of Lipschitz functions, Proc. Amer. Math Soc. 17 (1966), 665-669.

\section{ACKNOWLEDGMENT}

The preparation of the following two papers, which appeared in the May, 1966 issue of the Bulletin of the American Mathematical Society, was supported by the National Science Foundation under grant NSF G-16428 to the American Mathematical Society.

Whitehead torsion, by J. Milnor

Singular integrals, by A. P. Calderón

This information was inadvertently omitted from the BuLLETIN. 